\title{
Systematics of Ariantinae (Gastropoda, Pulmonata, Helicidae), a new approach to an old problem
}

\author{
Dick S.J. Groenenberg ${ }^{1,4}$, Peter Subai ${ }^{2}$, Edmund Gittenberger ${ }^{1,3}$ \\ ${ }^{1}$ Naturalis Biodiversity Center, P.O. Box 9505, Sylviusweg 70, 2333 BE Leiden, The Netherlands \\ ${ }^{2}$ Kronenberg 143, D-52074, Aachen, Germany \\ ${ }^{3}$ Institute of Biology, Leiden University, P.O. Box 9505, 2300 RA Leiden, The Netherlands \\ ${ }^{4}$ E-mail: Dick.Groenenberg@naturalis.nl
}

Key words: Ariantinae, classification, molecular phylogeny, morphology

\begin{abstract}
A new starting-point in Ariantinae systematics is presented by combining data on traditional shell morphology and genital anatomy, with phylogeny reconstructions based on DNA sequence data. For nearly all genera and subgenera one or more shells are depicted and drawings of the proximal part of the genital organs are shown to illustrate the morphological diversification within the subfamily. For as much as our material allowed it, partial sequences are presented for Histone $H 3$ (H3), Cytochrome c oxidase subunit I (COI), Cytochrome B (CytB) and $16 S$ ribosomal RNA (16S). Some of the allegedly speciose genera like Chilostoma and Campylaea (Zilch, 1960) do not represent monophyletic groups of species, whereas most of the remaining nominal taxa (e.g. Causa, Dinarica, Josephinella, Faustina, Liburnica, Kosicia and Thiessea) warrant a separate taxonomic status indeed. Sequence data from individual markers were informative at the species-level, but not for higherlevel phylogenetics. Insight in genus-level relationships was obtained after concatenation of the individual datasets. The Ariantinae are estimated to have originated during the late Cretaceous (Campanian), not later than ca. 80 million years ago. The enigmatic and morphologically aberrant, monotypic genus Cylindrus is shown as the sister-group of Arianta, a genus including A. arbustorum, which is also unusual in shell-shape and habitat. Ariantopsis and Wladislawia are classified as subgenera of neither Campylaea nor Chilostoma, but Cattania. Sabljaria is considered a subgenus of Dinarica. The nominal genus Superba is shown to be paraphyletic; additional data should demonstrate whether Superba has to be synonymised with Liburnica. The Ariantinae are here divided in 21 genera (2 new) and 13 subgenera (3 new).
\end{abstract}

\section{Contents}

Introduction …….................................................................. 37

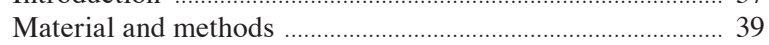

Taxon sampling ................................................................. 39

Genital anatomy ................................................................ 39

DNA extraction, PCR and sequencing ............................ 39

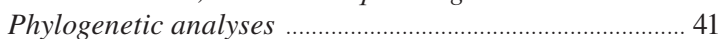

$\quad$ Fossil occurrences and age of taxa
$\quad$ Genetic distances
Systematics
Results
Discussion
Acknowledgements
References

\section{Introduction}

The classification of the Ariantinae Mörch, 1864 (Gastropoda, Helicidae), a subfamily of terrestrial airbreathing snails, with a primary radiation in southern Europe, has been under debate for more than a century. Apart from a few exceptions such as Cylindrus obtusus, Helicigona lapicida and Isognomostoma isognomostomos, most species within this subfamily are conchologically close to a basic bauplan (see Appendix), with shells that are more or less depressed globular, with an open umbilicus and no apertural teeth. All species are characterized by a pair of accessory glands in the genital system, inserting between the dart sac (= bursa telae) and the bursa copulatrix. In the literature, these glands are often referred to as mucous glands, a term that should be preferentially used for glands in the snail's foot-sole, however. The accessory glands can either be undivided or more or less completely split and are always longer than the dart sac.

The conchological uniformity did not hamper the description of new species, and higher taxa, what made the delimitation of genera and subgenera increasingly subjective. In the literature, more than once, taxon status was changed from a generic to a subgeneric level, or the other way round, without proper argumentation. This led to the confusing situation of today, where 
there is neither consensus on the identification and ranking of the taxa that should be accepted within the Ariantinae, nor about their mutual phylogenetic relationships.

Thus, the main issue regarding the systematics of this subfamily is not the delimitation of species, but the distinction between genera and subgenera. This is illustrated by the number of species-group taxa that have been described after Mörch (1864) erected the subfamily, and even more so by the number of genera used by different authors to classify the same species. Ariantopsis pelia, for example, has also been classified in Arianta, Chilostoma, Faustina, and Helicigona. In the taxonomic literature a variety of subdivisions of the Ariantinae has been proposed (e.g. Sturany and Wagner, 1914; Hesse, 1931; Knipper, 1939; Zilch, 1960; Subai, 1984, 1996; Bank et al., 2001; Subai, 2002; Subai and Fehér, 2006; Schileyko, 2006, 2013), among which the enumeration by Zilch (Table 1) has been most frequently cited. The classification resulting from this study will be compared in some detail with only the latter. This article is an extended version of a publication by Groenenberg et al. (2012), which was published only as a part of a doctoral thesis. In a recent article Cadahia et al. (2013) published similar data on the phylogeny of the Ariantinae, dealing with fewer taxa, however, and without discussing the implications for classification and nomenclature. Schileyko (2006, 2013) suggested classifications of the Ariantinae on the basis of morphological data. Initially (Schileyko, 2006) Marmorana Hartmann, 1844, with some generally accepted close relatives, and Theba Risso, 1826, were considered to belong to the Ariantinae. Later on, however (Schileyko, 2013), these genera were classified in other subfamilies, viz. Murellinae Hesse, 1918 and Thebinae Wenz, 1923. Interestingly, on the basis of a preliminary DNA analysis, using $\mathrm{COI}$ sequences in GenBank, Marmorana, Murella, and Tyrrheniberus showed up as Ariantinae indeed, whereas Theba has to be excluded as a genus of that subfamily. Thus, the status of the so-called Murellinae has to be studied in more detail.

Table 1. Selection of former classifications of the Ariantinae by different authors.

\begin{tabular}{|c|c|c|c|c|c|}
\hline \multicolumn{2}{|c|}{ Sturany and Wagner (1914) } & \multicolumn{2}{|l|}{ Zilch (1960) } & \multicolumn{2}{|c|}{ Bank et al. (2001) 'Clecom' } \\
\hline Genus & Subgenus & Genus & Subgenus & Genus & Subgenus \\
\hline \multirow[t]{4}{*}{ Campylaea } & Cattania & Arianta & & Arianta & \\
\hline & Campylaea & Campylaea & Ariantopsis & Causa & \\
\hline & Dinarica & & Campylaea & Chilostoma & Ariantopsis \\
\hline & Liburnica & & Delphinatia & & Campylaea \\
\hline Cylindrus & & & Dinarica & & Campylaeopsis \\
\hline \multirow[t]{6}{*}{ Helicigona } & Arianta & & Faustina $(=$ Cattania $)$ & & Cattania \\
\hline & Campylaeopsis & & Liburnica & & Chilostoma \\
\hline & Cingulifera & & Wladislawia & & Cingulifera \\
\hline & Drobacia & Chilostoma & Campylaeopsis & & Corneola \\
\hline & Helicigona & & Chilostoma & & Delphinatia \\
\hline & Thiessea & & Cingulifera & & Dinarica \\
\hline Isognomostoma & & & Drobacia & & Josephinella \\
\hline \multirow{10}{*}{ Vidovicia } & & & Josephinella & & Kosicia \\
\hline & & & Kosicia & & Liburnica \\
\hline & & & Thiessea & & Thiessea \\
\hline & & Cylindrus & & & Wladislawia \\
\hline & & Helicigona & & Cylindrus & \\
\hline & & Isognomostoma & & Drobacia & \\
\hline & & Vidovicia & & Faustina & \\
\hline & & & & Helicigona & \\
\hline & & & & Isognomostoma & \\
\hline & & & & Vidovicia & \\
\hline 5 genera & 10 subgenera & 7 genera & 14 subgenera & 9 genera & 14 subgenera \\
\hline
\end{tabular}




\section{Material and methods}

\section{Taxon sampling}

This study is based on 172 specimens (including 5 outgroup specimens) from 85 (sub)species of Ariantinae from across Europe, representing about half of the known species and all the currently accepted genera (see Appendix). Specimens were collected in the period 1957-2012 and most material was fixed and conserved in $70 \%$ ethanol or isopropanol. Some (old) specimens were stored in "spiritus" (methylated spirits), whereas the more recently collected specimens were preserved in $97 \%$ ethanol.

\section{Genital anatomy}

For nearly all the (sub)genera the genital tract is illustrated (see Appendix). These figures are arranged according to the type of accessory glands, i.e. undivided versus one or both glands split. Only Josephinella vikosensis, with undivided accessory glands, was illustrated next to two congeneric species with split glands. For all the (sub)genera our personal observation regarding the accessory glands is presented. We refrained from an analysis of all the data that can be found in the literature (often without information on the actual number of individuals that was investigated). For the ease of comparison we only differentiate between undivided vs. split accessory glands, i.e. specimens in which only one of the glands was divid- ed, as well as those with trifurcate glands, were considered split.

\section{DNA extraction, PCR and sequencing}

Total genomic DNA was extracted from small foot tissue samples using a DNeasy Tissue Kit (Qiagen) following the manufacturer's instructions. As a follow-up of an earlier investigation (Gittenberger et al., 2004), this study started with the amplifcation of COI, but due to the poor quality of some of the DNA extracts, mini-barcode primers (Hajibabaei et al., 2006; Meusnier et al., 2008) were occasionally used to amplify a smaller fragment of COI (124 bp fragment excluding primersites). These mini-barcode sequences grouped with those of conspecifics, or otherwise with congeners, for which the $655 \mathrm{bp} C O I$ fragment was obtained. Although most of the recognized (sub)genera formed well supported clades (based on Bayesian phylogeny inference), the relationships between the (sub)genera were poorly supported. Therefore nuclear marker $H 3$ and mitochondrial markers $C y t B$ and $16 S$ were added. PCR primers and references are given in Table 2. PCRs were carried out in $25 \mu \mathrm{l}$ volumes using 1.25 units of Taq DNA polymerase (Qiagen), $0.4 \mathrm{mM}$ of each primer and $0.2 \mathrm{mM}$ dNTPs. For $C O I$ the final $\mathrm{MgCl}_{2}$ concentration occasionally had to be increased to $2.5 \mathrm{mM}$ (1x PCR buffer contains $1.5 \mathrm{mM}$; Qiagen). For 16S, Q-solution (Qiagen; final concentration $1 \times$ ) was added to most of the reactions. PCR thermoprofile: inititial denaturation $3 \mathrm{~min}$. @ $94^{\circ} \mathrm{C}$, followed by

Table 2. Primer information. * Amplicon length excluding primer sequence. ${ }^{* *}$ Annealing temperature. ${ }^{* * *}$ Minibarcode Reverse primer has been modified to be more specific for Ariantinae.

\begin{tabular}{|c|c|c|c|c|c|}
\hline Primer name & Sequence (5' to $\left.3^{\prime}\right)$ & Marker & Length $^{*}$ & Source & $\mathrm{AT}^{* *}$ \\
\hline H3-F & ATGGCTCGTACCAAGCAGACVGC & \multirow[t]{2}{*}{$\mathrm{H} 3$} & \multirow[t]{2}{*}{328} & \multirow[t]{2}{*}{ Colgan, 2000} & \multirow[t]{2}{*}{57} \\
\hline $\mathrm{H} 3-\mathrm{R}$ & ATATCCTTRGGCATRATRGTGAC & & & & \\
\hline L1490 & GGTCAACAAATCATAAAGATATTGG & \multirow[t]{2}{*}{$\mathrm{COI}$} & \multirow[t]{2}{*}{655} & \multirow[t]{2}{*}{ Folmer, 1994} & \multirow[t]{2}{*}{$45-50$} \\
\hline H2198 & TAAACTTCAGGGTGACCAAAAAATCA & & & & \\
\hline MB-F & TCCACTAATCACAARGATATTGGTAC & \multirow[t]{2}{*}{ COI mini-barcode } & \multirow[t]{2}{*}{124} & \multirow[t]{2}{*}{ Meusnier, 2008} & \multirow[t]{2}{*}{50} \\
\hline $\mathrm{MB}-\mathrm{R}^{* * * *}$ & GAAAATTATKACAAARGCATGAGC & & & & \\
\hline $151-\mathrm{F}$ & TGTGGRGCNACYGTWATYACTAA & \multirow[t]{2}{*}{ CytB } & \multirow[t]{2}{*}{361} & \multirow[t]{2}{*}{ Merritt, 1998} & \multirow[t]{2}{*}{50} \\
\hline $270-\mathrm{R}$ & AANAGGAARTAYCAYTCNGGYTG & & & & \\
\hline Pal-F & CGGCCGCCTGTTTATCAAAAACAT & \multirow[t]{2}{*}{$16 \mathrm{~S}$} & \multirow[t]{2}{*}{$404-412$} & \multirow[t]{2}{*}{ Palumbi, 1991} & \multirow[t]{2}{*}{50} \\
\hline Pal-R & GGAGCTCCGGTTTGAACTCAGATC & & & & \\
\hline
\end{tabular}


Table 3. Information content per dataset. Inf. char. $=$ The number of parsimony informative characters. Perc. inf. $=$ The percentage of informative characters, calculated as $100 \times$ (Inform. char. / Total char.).

\begin{tabular}{lccccc}
\hline Dataset & Specimens & Total char. & Constant char. & Inf. char. & Perc. inf. \\
\hline H3 & 161 & 328 & 266 & 46 & 14.0 \\
COI & 149 & 655 & 339 & 300 & 45.8 \\
CB & 91 & 361 & 128 & 222 & 61.5 \\
16S & 82 & 335 & 163 & 149 & 44.5 \\
H3-COI-CB & 89 & 1344 & 748 & 558 & 41.5 \\
H3-COI-CB- $16 S$ & 103 & 1679 & 912 & 711 & 42.3 \\
\hline
\end{tabular}

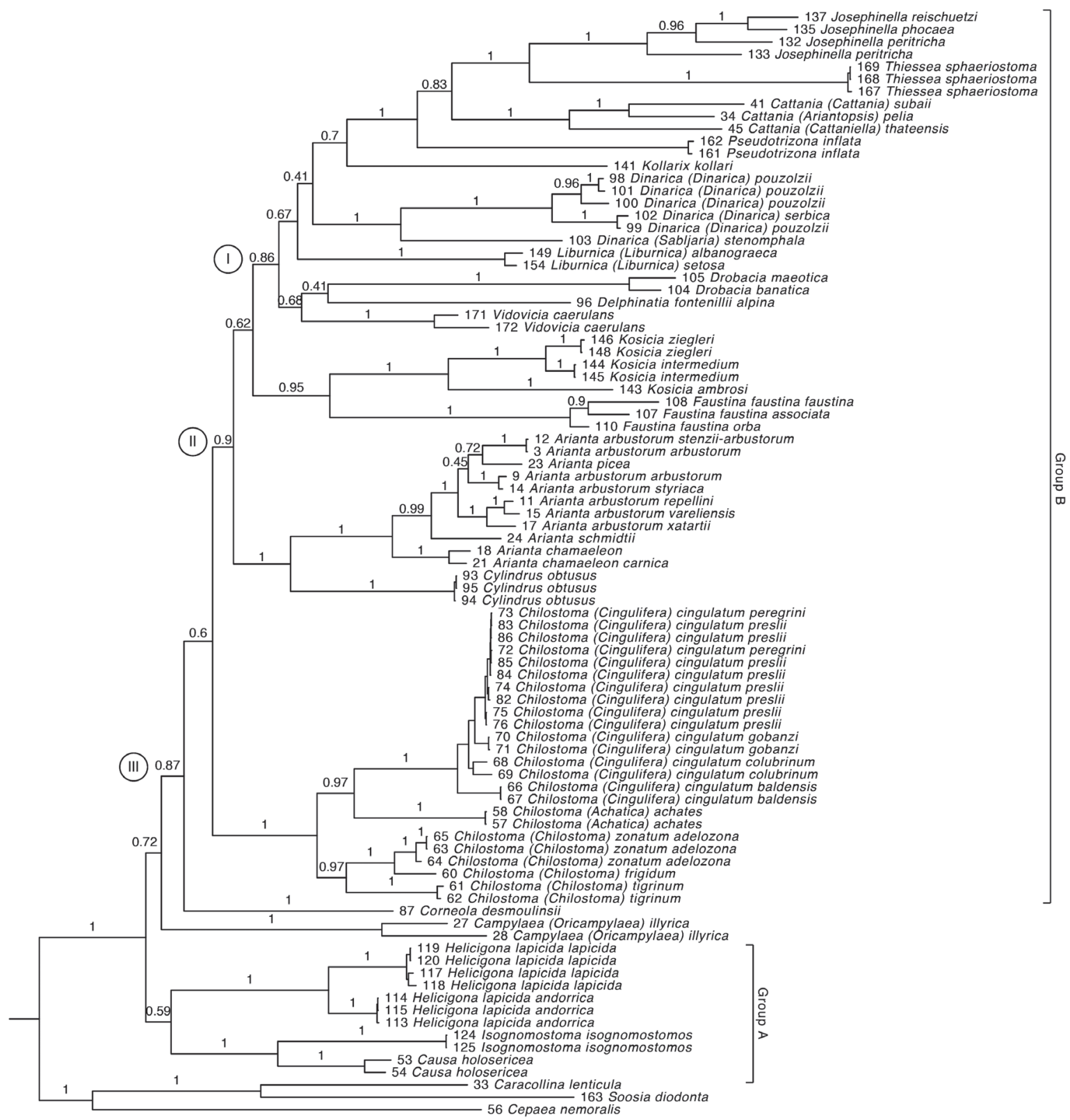

Fig. 1. MrBayes phylogeny based on the 'stringent' $\mathrm{H3}-\mathrm{COI}-\mathrm{CytB}$ dataset. Branch values show posterior probabilities. 
40 cycles of - denaturation $15 \mathrm{sec} @ 94^{\circ} \mathrm{C}$, annealing 30 sec. @ AT (Table 2), extension 40 sec. @ 72 ${ }^{\circ} \mathrm{C}-$ and a final extension of $5 \mathrm{~min}$. $972^{\circ} \mathrm{C}$. PCR products were cleaned with a Montage purification kit (Millipore) at Macrgoen Inc. Europe (Amsterdam), where they were sequenced in both directions on an $\mathrm{ABI} 37730 \mathrm{XL}$ using the same primers as used for the PCR. Forward and reverse sequences were assembled with Sequencher 4.10.1 (Gene Codes Corporation) and protein coding genes $(\mathrm{H} 3, \mathrm{COI}$ and $\mathrm{Cyt} \mathrm{B})$ were manually aligned in MacClade 4.08 (Maddison and Maddison, 2005). The alignment for $16 \mathrm{~S}$ was made with MAFFT (Katoh and Standley, 2013) as implemented in the software package Geneious Pro 7.0.6 using the default settings with the G-INS-i algorithm. Nonconserved blocks of sequence data were removed from the alignment with Gblocks (Castresana, 2002) conducted on the Gblocks Server (http://molevol.cmima. csic.es/castresana/Gblocks_server.html), using only the 'more stringent selection' option (which restricts the introduction of contiguous nonconserved positions).

\section{Phylogenetic analyses}

For each dataset (each marker) a nucleotide substitution model was selected with MrModeltest 2.2 (Nylander, 2004). For the mitochondrial datasets the model was GTR+I+G, for $H 3$ it was HKY+I+G. Bayesian analyses were done in MrBayes 3.2.1. (Ronquist and Huelsenbeck, 2003) hosted on the CIPRES Science Gateway (Miller, 2010). For each marker the analysis consisted of two simultaneous, four chain, MCMC runs (10 M generations). Trees were sampled every 1000 generations, the first 2500 trees were discarded as burnin (relburnin $=$ yes, burninfrac $=0.25$ ) . Examination of the .p output files in Tracer v.1.5 (Rambaut and Drummond, 2007) showed stationarity was reached with proper effective sample sizes for all parameters (ESS > 200). Sumtrees (Sukumaran and Holder, 2010) was used to calculate $25 \%$ majority rule consensus trees. Subsequently the datasets for the individual markers were combined into two concatenated datasets (from hereon referred to as): the 'stringent' and 'relaxed' datasets. The stringent dataset ( 89 taxa) consisted of only protein coding genes (i.e. H3, COI and $C y t B)$ and had no missing data. The relaxed dataset (103 taxa) consisted of all markers (H3, COI, CytB and $16 S$ ); taxa for which only one marker was missing were also included. A partitioned analysis was set up in MrBayes (same version) for both datasets; for each partition the GTR $+\mathrm{I}+\mathrm{G}$ model was selected using the above described procedure.

\section{Fossil occurrences and age of taxa}

In a recent check-list of fossil land snails of western and central Europe, Nordsieck (2014) reviews fossil taxa based on stratigraphic ranges. The oldest and only indisputably identified Ariantinae fossil in that list is of a Helicigona species from the late Burdigalian, Early Miocene (17.5-16 MYA; references in Nordsieck, 2014). This fossil was used as a single calibration point imposing a normal distribution prior (mean 16.75 MY, stdev 0.375) allowing for soft minimum and maximum age boundaries. Inital BEAST analyses were performed with and without setting the monophyly of the Ariantinae sensu auct. as a constraint. Species of the genera Cepaea, Caracollina and Soosia were used as outgroup taxa that are traditionally classified in closely related taxa within the same superfamily Helicoidea. If the monophyly of the Ariantinae sensu auct. was not set as a constraint, many internodes appeared between these outgroup taxa and the root of the tree. Re-rooted with the outgroup taxa, the topology was virtually identical to the ML (not shown) and MrBayes phylogenies. In our BEAST analyses, clades I, II and III (PP $\geq 0.86$ ) were therefore used as a constraint. Three runs consisting of $100 \mathrm{M}$ generations were performed (for both the stringent and relaxed datasets) using a relaxed clock model (lognormal uncorrelated) and with the Yule process (Yule, 1924; Gernhard, 2008) set as tree prior (BEAUti; Drummond et al., 2012). After initial inspection with Tracer v.1.5 (Rambaut and Drummond, 2007), for each dataset the log and tree files were combined with Logcombiner v.1.7.5 (Rambaut and Drummond, 2007) disregarding $10 \mathrm{M}$ generations $(10 \%)$ as burnin. ESS values were all above 200. Subsequently TreeAnnotator v.1.7.5 (Rambaut and Drummond, 2007) was used (burnin set to zero) to generate the maximum clade credibility tree for both the combined tree files. Given the use of a single calibration point and the overall low posterior probabilities, the obtained ages should be considered indicative only. Confidence intervals (node bars) were so large (especially for the deeper nodes) that they obscured the tree and hence were omitted for clarity. Node ages were rounded to the first decimal to still visualize the differences in results between 'stringent' and 'relaxed' dataset, not to imply accuracy. 


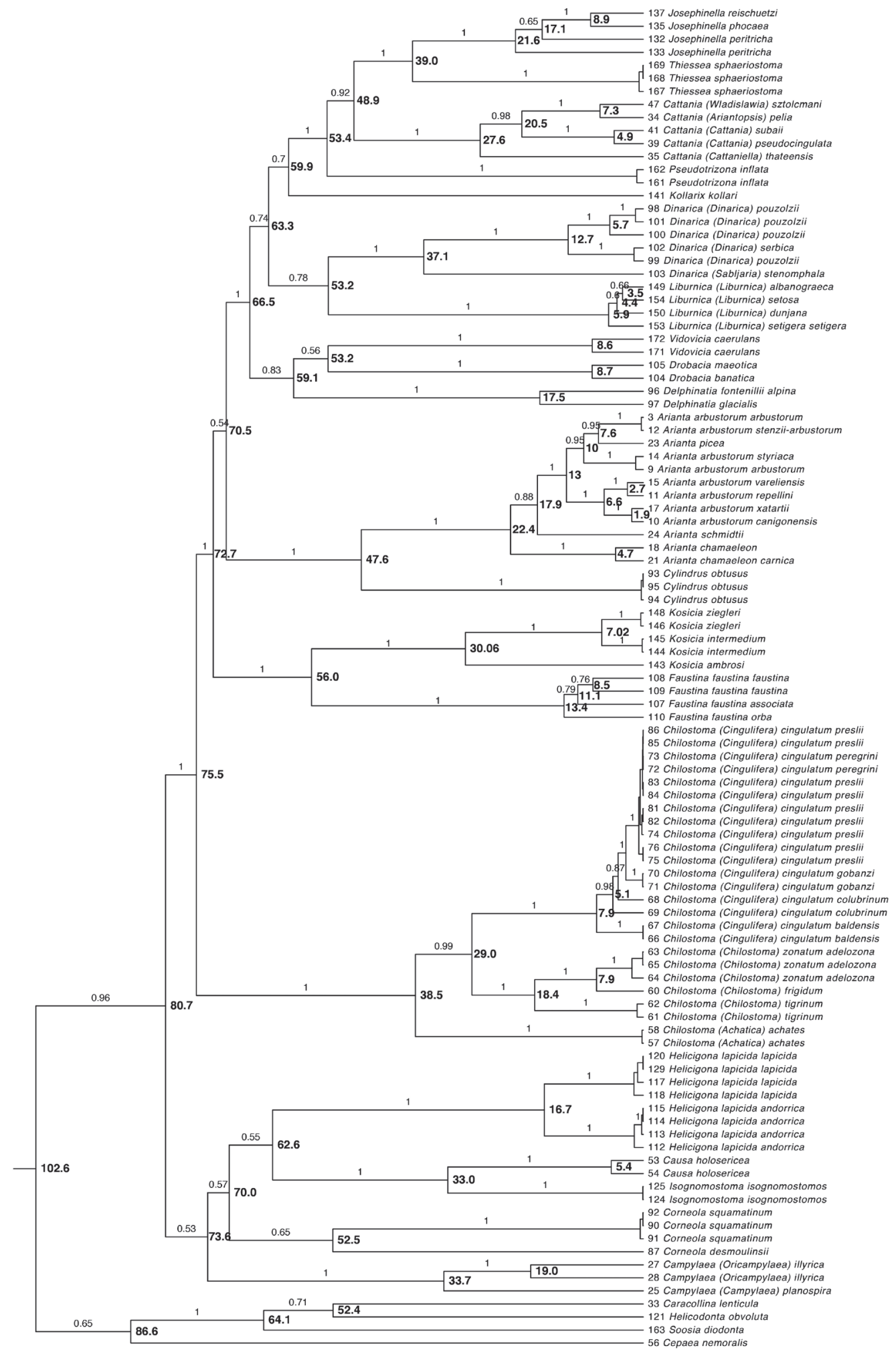

Fig. 2. BEAST phylogeny based on the 'relaxed' H3-COI-CytB-16S dataset. Branch values show posterior probabilities. Node values indicate divergence estimates in MYA. 


\section{Genetic distances}

Uncorrected pairwise $(p)$ distances were calculated with Paup 4.0b10 for Unix (Swofford, 2002) for each protein coding gene. Sequence divergence percentages were calculated as the uncorrected $p$-distance times 100.

\section{Systematics}

Genera and subgenera are taxonomic ranks. To make the ranking less subjective, a cladistic approach is required. Throughout this study, clades that are sistergroups of one another do not differ in their taxonomic ranking. This leaves the option open to give the same ranking also to clades that do not have a sister-group relationship. As a consequence, a genus may have more than two subgenera. Since there are many more splitting points in evolutionary history than taxonomic ranks, this cannot be avoided. Genera are always based on monophyletic species groups. In some cases, at the subgeneric level, paraphyletic taxa are accepted. Genetic distances were not used as decisive in deciding upon the status of genus versus subgenus.

When morphologically cryptic taxa are unequivocally brought to light by the molecular analyses, these taxa are not neglected but formally characterized and named, as advocated by Cook et al. (2010), Gittenberger and Gittenberger (2011) and Jörger and Schrödl (2013). Abbreviations: PP = posterior probability, MYA = million years ago.

\section{Results}

In total 483 sequences were obtained: 161, 149, 91 and 82 for the markers $H 3, C O I, C y t B$ and $16 S$, respectively. A summary of the character statistics (as calculated with Paup 4.0b10 for Unix; Swofford, 2002) for each dataset is given in Table 3. Table 4 (Appendix) gives a summary of uncorrected $p$-distances.

Both the phylogeny reconstructions for the individual markers (Figs S1-S4; online supplementary information), as well as those for the concatenated datasets (Figs 1, 2, S5, S6) distinguish most of the (sub)genera that were traditionally characterized by subtle conchological differences and geographic origin (e.g. Arianta, Cattania, Corneola, Chilostoma, Cingulifera, Dinarica, Faustina, Helicigona, Josephinella, Kosicia and Liburnica). Although sister-group relationships between some taxa were shown explicitly (e.g. Jose- phinella - Thiessea, Ariantopsis - Wladislawia), deeper nodes were hardly supported, particularly in the phylogenies based on the individual markers (Figs S1-S4). The $H 3$ dataset differs most from the other datasets by its relatively low percentage of parsimony informative characters (Table 3). The phylogeny based on this marker is not discriminative below the genus level, but can be useful for the assignment of species (or subgenera) to genera (e.g. Ariantopsis pelia, Campylaeopsis moellendorffii, Superba spec., Wladislawia sztolcmani). The Histone gene cluster is multicopy (slight intercopy variation might exist and $H 3$ pseudogenes have been reported; Rooney et al., 2002) and has been used in higher-level phylogenetics (Armbruster et al., 2005; Colgan et al., 2007 and references therein). For some species of mostly Chilostoma, double peaks were observed at a few positions within the $H 3$ sequence. This genus which is shown as monophyletic in nearly all phylogeny reconstructions (Figs 1, 2, S2, S3, S5 and S6), turns out paraphyletic in the phylogeny for $H 3$ (Fig. S1). It might be argued that the more extensive sampling of Chilostoma (Cingulifera) increased the chance of observing this apparent intercopy variation, but it was not observed in other genera for which multiple species were sequenced (e.g. Arianta, Cattania, Josephinella and Liburnica) either. In agreement with Colgan et al. (2000) we therefore conclude that intercopy variation in $H 3$ will not significantly interfere with the phylogeny reconstructions.

Initially no amplicons were obtained with the $\mathrm{COI}$ mini-barcode primers of Meusnier et al. (2008); to get these working for Ariantinae, the reverse primer was modified (Table 2). Hajibabaei et al. (2006) showed (in silico) that COI mini-barcodes (109 bp; compared to the full length barcode of $654 \mathrm{bp}$ ) are $3 \%$ less effective in the correct identification of closely related species and pointed out that mini-barcodes might be less useful for the classification of specimens in larger species assemblages. Based on those taxa for which both a complete and a mini-barcode sequence were obtained (C. (Cattania) faueri, Corneola desmoulinsii, Helicigona lapicida andorrica and Vidovicia caerulans), we conclude that the mini-barcodes (despite their short length) are placed correctly in the COI phylogeny (Fig. S2).

The phylogeny reconstructions for the concatenated datasets (Figs 1, 2, S5-S6) show a basal split within the Ariantinae, differentiating the ancestor of the genera Causa, Isognomostoma and Helicigona, referred to here as group A, from that of all other genera (except Campylaea and Corneola), collectively referred to as 
group B. It remains unclear whether Campylaea and Corneola belong to A (Fig. 2) or B (Figs 1, S5), or should be considered a group on their own (Fig. S6).

All of the obtained sequences were deposited in GenBank; a complete list of taxa, sampling information and accession numbers is given in Table S7.

\section{Discussion}

Left aside some exceptions, Ariantinae shells are rather monomorphic (Fig. 3, Appendix). Because of the limited number of conchological characters, many authors studied the genital tract for morphological characters that could discriminate species and especially higher taxa. However, the genital morphology within this subfamily is also very homogeneous, what is uncommon among pulmonates. The form of the accessory glands, which are either undivided or more or less completely split (Fig. 4, Appendix), has been used by some authors as a (partial) basis for the systematics of the Ariantinae (Sturany and Wagner, 1914; Schileyko, 2006), although according to other authors both types of accessory glands can occur within the same genus, or even species (Hesse, 1931; Knipper, 1939; Schileyko, 2013). A classification of the species of Ariantinae in only two genera, as for example Helicigona and Campylaea (sensu Sturany and Wagner, 1914; Table 1), or Chilostoma and Campylaea (sensu Zilch, 1960; Table 1) is an oversimplification according to all modern authors, but what classification should be accepted alternatively remains a matter of dispute. Recently it has been suggested that the structure of the penial papilla might be a useful character to clarify the phylogenetic relationships between the (sub)genera within the subfamily (Schileyko, 2013), but that view still has to be confirmed.

Obviously, given the actual situation, a new approach is necessary, as was realized most recently by Groenenberg et al. (2012) and Cadahia et al. (2013), who tried to escape from the confusion by the use of molecular phylogenetics. Despite its shortcomings in the completeness of the molecular data, this article expands the reliability of the molecular phylogeny reconstructions, enabling a still better founded discussion regarding the subdivisions of the Ariantinae.

Our analyses do not support an evolutionary significance of the transformation series based by Schileyko (2013) on the structure of the penial papilla in several Ariantinae genera. Dinarica and Cattania are not closely related to Helicigona, for example, so that the depicted morphocline Cattania - Helicigona - Dinarica
(Schileyko, 2013) cannot be interpreted in an evolutionary context.

Aiming at a general classification of the Ariantinae, the shape of the accessory glands is equally uninformative. The transition from undivided to split gland(s), or the other way round, must have occurred more than once.

When the phylogeny reconstructions obtained with this study are compared to generic classifications based on conchology and geography, nearly all the named (sub)genera are recovered as distinct clades. A few additional (sub)generic groups were discovered and described, viz. Campylaea (Oricampylaea), Chilostoma (Achatica), Cattania (Cattaniella), Pseudotrizona, Kollarix (Table 5, Appendix). The phylogenetic relationships above the genus-level, as indicated by the lower posterior probabilities, remain less certain in many cases. For a limited number of genera, sister-group relationships were disclosed, i.e. AriantaCylindrus, Causa-Isognomostoma, JosephinellaThiessea and Kosicia-Faustina (PP $\geq 0.95$; Figs 1, 2, S5, S6). In particular the close relationship between Cylindrus and Arianta is intriguing. Clearly both genera are part of a lineage that was less restricted in the development of conchological novelties. The classification of Cylindrus as a member of the Ariantinae is now confirmed genetically.

We agree with Cadahia et al. (2013) that indications of evolutionary age are uncertain, to say the least. The fossil record is very incomplete indeed, and a molecular clock model is also not easily applicable. The unattractive alternative would have been to omit such speculations altogether.

\section{Acknowledgements}

We thank all the colleagues who put specimens at our disposal (Table S7). We are indebted to H.A. Thomassen and R. Glas (Leiden) for some of the labwork carried out in the early stages of this project. Finally we like to thank Dr. Neubert (Dr. E. Neubert (Bern), Dr. A.A. Schileyko (Moscow) and two anonymous reviewers for their remarks and constructive comments on an earlier version of this manuscript.

\section{References}

Armbruster GFJ, Böhme M, Bernhard D, Schlegel M. 2005. The H3/H4 histone gene cluster of land snails (Gastropoda: Stylommatophora): TS/TV ratio, GC3 drive and signals in stylommatophoran phylogeny. Journal of Molluscan Studies 71: 339-348. doi: 10.1093/mollus/eyi038 
Bank RA. 2012. Fauna Europaea version 2.4: Mollusca. http:// www.faunaeur.org

Bank RA, Bouchet P, Falkner G, Gittenberger E, Hausdorf B,Von Proswitz T, Ripken TEJ. 2001. Supraspecific classification of European non-marine Mollusca (CLECOM Sections I+II). Heldia 4: 77-128.

Beck H. 1837. Index molluscorum præsentis ævi musei principis augustissimi Christiani Frederici. Hafniae, Copenhagen.

Bielz M. 1851. Verzeichniss der Land- und Süsswasser-Mollusken Siebenbürgens. Verhandlungen und Mittheilungen des Siebenbürgischen Vereins für Naturwissenschaften in Hermannstadt 2: 14-16, 55-59, 62-65.

Boschi C. 2011. Die Schneckenfauna der Schweiz. Haupt Verlag, Bern, Stuttgart, Wien.

Bourguignat JR. 1857. Aménités malacologiques. Revue et Magasin de Zoologie pure et appliquée (2) 9: 3-21, Pl. 1-4, 8-10.

Bourguignat JR. 1870. Mollusques nouveaux, litigieux ou peu connus. Revue et Magasin de Zoologie pure et appliquée et de Sériciculture Comparée (2) 22: 14-30, 87-97, 166-171, pl. 14-17. Paris.

Bourguignat JR. 1878. Species novissimae molluscorum in Europaeo systemati detectae, notis diagnosticis succinctis breviter descriptae. Klincksieck, Paris.

Bourguignat JR. 1880. Description de quelques espèces de mollusques terrestres et fluviatiles des environs de Saint-Martin-de-Lantosque (Alpes-Maritimes). Vidal, Cannes.

Brusina S. 1904. Zur Rettung unserer Mollusken-Fauna. Nachrichtsblatt der Deutschen Malakozoologischen Gesellschaft 36: 157-168.

Cadahía L, Harl J, Duda M, Sattmann H, Kruckenhauser L, Fehér Z, Zopp L, Haring E. 2013. New data on the phylogeny of Ariantinae (Pulmonata, Helicidae) and the systematic position of Cylindrus obtusus based on nuclear and mitochondrial DNA marker sequences. Journal of Zoological Systematics and Evolutionary Research. doi: 10.1111/jzs.12044

Castresana J. 2002. GBLOCKS: selection of conserved blocks from multiple alignments for their use in phylogenetic analysis, Version 0.91b.

Colgan DJ, Ponder WF, Eggler PE. 2000. Gastropod evolutionary rates and phylogenetic relationships assessed using partial 28S rDNA and histone H3 sequences. Zoologica Scripta 29: 29-63.

Colgan DJ, Ponder WF, Beacham E, Macaranas J. 2007. Molecular phylogenetics of Caenogastropoda (Gastropoda: Mollusca). Molecular Phylogenetics and Evolution 42: 717-737.

Cook LG, Edwards RD, Crisp MD, Hardy NB. 2010. Invertebrate Systematics 24: 322-326.

Damjanov S, Likharev I. 1975. Fauna Bulgarica, Gastropoda terrestria, vol. IV. Publishing house of the Bulgarian Academy of Sciences, Sofia.

De Cristofori J, Jan G. 1832. Catalogus in IV sectiones divisus rerum naturalium in museo existantium Josephi De Cristofori et Georgii Jan. Sectio II Conchyliologia. Pars I. Conspectus methodicus Molluscorum I. Testacea terrestria et fluviatilia. Parmae.

Dedov IK. 1998. Annotated check-list of the Bulgarian terrestrial snails (Mollusca, Gastropoda). Linzer Biologische Beiträge 30/2: 745-765.

Deshayes GP. 1830. Encyclopédie méthodique. Histoire naturelle des vers. Tome second. Agasse, Paris.
Draparnaud JPR. 1801. Tableau des mollusques terrestres et fluviatiles de la France. Renaud; Bossange, Masson and Besson, Montpellier, Paris.

Draparnaud JPR. 1805. Histoire naturelle des mollusques terrestres et fluviatiles de la France. Ouvrage posthume. Avec XIII planches. Plassan, Paris; Renaud, Montpellier.

Drummond AJ, Suchard MA, Xie D, Rambaut A. 2012. Bayesian phylogenetics with BEAUti and the BEAST 1.7 Molecular Biology and Evolution 29: 1969-1973. doi: 10.1093/molbev/mss075

Falkner G. 1998. Malakologische Neufunde und Forschungsprobleme in den Bayerischen Alpen und ihrem Vorland. Pp. 89-124 in: Jung WH, ed., Naturerlebnis Alpen. Verlag Dr. Friedrich Pfeil, München.

Falkner G, Ripken TEJ, Falkner M. 2002. Mollusques continentaux de France. Liste de référence annotée et bibliographie. Patrimoines naturels 52: 1-350.

Farines JN. 1834. Description de trois espèces nouvelles de coquilles vivantes du département des Pyrénées-Orientales. J.-B Alzine, Perpignan.

Fauer W. 1991. Eine neue Chilostoma aus Griechisch-Ostmakedonien (Gastropoda Pulmonata: Helicidae). Archiv für Molluskenkunde 120: 167-170.

Férussac A. 1821-1822. Tableaux systématiques des animaux mollusques classés en familles naturelles, dans lesquels on a établi la concordance de tous les systèmes; suivis d'un prodrome général pour tous les mollusques terrestres ou fluviatiles, vivants ou fossiles. Bertrand, Paris; Sowerby, Londres.

Férussac A, Deshayes GP. 1819-1851. Histoire naturelle générale et particulière des mollusques terrestres et fluviatiles, tant des espèces que l'on trouve aujourd'hui vivantes, que des dépouilles fossiles de celles qui n'existent plus; classés d'après les caractères essentiels que présentent ces animaux et leurs coquilles. J-B Bailliere, Paris.

Fischer P. 1877. Faune malacologique de la vallée de Cauterets - Additions et corrections. Journal de Conchyliologie 25: 49-56. Paris.

Fitzinger LI. 1833. Systematisches Verzeichniß der im Erzherzogthume Oesterreich vorkommenden Weichthiere, als Prodrom einer Fauna desselben. Beiträge zur Landeskunde Oesterreich's unter der Enns (Verein für vaterländische Geschichte Wien) 3: 88-122.

Folmer O, Black M, Hoeh W, Lutz R, Vrijenhoek R. 1994. DNA primers for amplification of mitochondrial cytochrome c oxidase subunit I from diverse metazoan invertebrates. Molecular Marine Biology and Biotechnology 3: 294-299.

Frank C. 2006. Plio-pleistozäne und holozäne Mollusken Österreichs 2. Pp. 397-861 in: Frank C, Grillitsch H, ed., Mitteilungen der prähistorischen Kommission. Verlag der Österreichischen Akademie der Wissenschaften, Wien 62.

Frauenfeld von G. 1867. Zoologische Miscellen. XII. Verhandlungen der kaiserlich-königlichen Zoologisch-Botanischen Gesellschaft in Wien 17: 775-784.

Gavetti E, Birindelli S, Bodon M, Manganelli G. 2008. Molluschi terrestri e d'acqua dolce della Valle di Susa. Museo Regionale di Scienze Naturali, Torino, Monografie 44: 1-274.

Geneious Pro v. 7.0.6 created by Biomatters. Available from: http://www.geneious.com.

Gernhard T. 2008. The conditioned reconstructed process. Journal of Theoretical Biology 253: 769-778. 
Gittenberger A, Gittenberger E. 2011. Cryptic, adaptive radiation of endoparasitic snails: sibling species of Leptoconchus (Gastropoda: Coralliophilidae) in corals. Organisms Diversity and Evolution 11: 21-41.

Gittenberger E, Piel WH, Groenenberg DSJ. 2004. The Pleistocene glaciations and the evolutionary history of the polytypic snail species Arianta arbustorum (Gastropoda, Pulmonata, Helicidae). Molecular Phylogenetics and Evolution 30: 64-73. doi:10.1016/S1055-7903(03)00182-9

Groenenberg DSJ, Subai P, Gittenberger E. 2012. Pp. 117-169 in: Molecular taxonomy and natural history collections. Chapter 6. PhD thesis. ISBN /EAN 9789461083470.

Haas F. 1936. Kurze Bemerkungen, VII. Archiv für Molluskenkunde 68: 127-131.

Haase M, Misof B. 2009. Dynamic gastropods: stable shell polymorphism despite gene flow in the land snail Arianta arbustorum. Journal of Zoological Systematics and Evolutionary Research 47: 105-114.

Hajibabaei M, Smith MA, Janzen DH, Rodriguez JJ, Whitfield JB, Hebert PDN. 2006. A minimalist barcode can identify a specimen whose DNA is degraded. Molecular Ecology Notes 6: 959-964.

Held F. 1837. Notizen über die Weichthiere Bayerns. (Fortsetzung.). Isis (12): 902-919.

Hesse P. 1912. Beschreibungen neuer Arten. Nachrichtsblatt der Deutschen Malakozoologischen Gesellschaft 44 (2): 56-62.

Hesse P. 1931. Zur Anatomie und Systematik palaearktischer Stylommatophoren. Zoologica 31: 1-118.

Jaeckel SGH. 1962. Ergänzungen und Berichtigungen zum rezenten und quartären Vorkommen der mitteleuropäischen Mollusken. In: A. Zilch and S.G.A. Jaeckel, Mollusken. Die Tierwelt Mitteleuropas 2 (1), Ergänzung: 25-294.

Jörger KM, Schrödl M. 2014. How to describe a cryptic species? Practical challenges of molecular taxonomy. Frontiers in Zoology 10: 59. doi: 10.1186/1742-9994-10-59.

Katoh K, Standley DM. 2013. MAFFT multiple sequence alignment software version 7: improvements in performance and usability. Molecular Biology and Evolution 30 (4): 772-780. doi: $10.1093 / \mathrm{molbev} / \mathrm{mst} 010$

Kerney MP, Cameron RAD. 1979. Field guide to the land snails of Britain and north-west Europe. Collins.

Kierdorf-Traut G. 2012. Notizen zur Verbreitung von Chilostoma cingulatum peregrini Falkner, 1998 in Nordtirol (Gastropoda: Helicidae). Gredleriana 12: 147-154.

Kimakowicz von M. 1890. Beitrag zur Molluskenfauna Siebenbürgens. II. Nachtrag. Verhandlungen und Mittheilungen des Siebenbürgischen Vereins für Naturwissenschaften in Hermannstadt 40: 1-113.

Knipper H. 1939. Systematische, anatomische, ökologische und tiergeographische Studien an südosteuropäischen Heliciden. (Moll. Pulm.). Archiv für Naturgeschichte (N.F.) 8: 327-517.

Kobelt W. 1871. Nachrichtsblatt der Deutschen Malakozoologischen Gesellschaft 3: 70-73.

Kobelt W. 1872. Neue oder wenig bekannte Campyläen. Malakozoologische Blätter 19: 125-133.

Kobelt W. 1876. Iconographie der Land- und Süsswasser-Mollusken mit vorzüglicher Berücksichtigung der europäischen noch nicht abgebildeten Arten von E. A. Rossmässler 4. Kreidel, Wiesbaden.

Kobelt W. 1902-1904. Iconographie der Land- und SüsswasserMollusken mit vorzüglicher Berücksichtigung der euro- päischen noch nicht abgebildeten Arten von E.A. Rossmässler. Neue Folge 9, 5-6: 57-86 [1902]; 10, 1-4: 1-48 [1903]; 11: i-xii, 1-342 [1904]. Kreidel, Wiesbaden.

Koene JM, Schulenburg H. 2005. Shooting darts: co-evolution and counter-adaptation in hermaphroditic snails. BMC Evolutionary Biology 5: 25.

Krejci K, Wenz W. 1926. Jungtertiäre Landschnecken aus Südrumänien. Neues Jahrbuch für Mineralogie, Geologie und Paläontologie 55 (B): 53-65.

Kroupa O. 1994. Zur Molluskenfauna Bulgariens. 3. Über Arianta pelia (Hesse, 1912) (Stylommatophora, Helicidae). Nachrichtenblatt der Ersten vorarlberger malakologischen Gesellschaft 2: 22-28.

Lamarck de Monet de JBPA. 1792. Sur quatre espèces d'hélices. Journal d'Histoire Naturelle 2: 347-353.

Lamarck de Monet de JBPA. 1822. Histoire Naturelle des Animaux sans Vertèbres. Chez l'auteur, Paris.

Linnaeus C. 1758. Systema Naturae per Regna Tria Naturae, Secundum Classes, Ordines, Genera, Species, cum Characteribus, Differentiis, Synonymis, Locis. Tomus I. Editio Decima, Reformata. Laurentii Salvii, Holmiae, Stockholm.

Maassen WJM. 1985. Helicigona kollari L. Pfeiffer, 1856. De Kreukel 21: 5-6.

Maddison DR, Maddison WP. 2005. MacClade 4.08. Sinauer Associates, Sunderland, MA.

Menke CT. 1830. Synopsis methodica molluscorum generum omnium et specierum earum, quae in museo Menkeano adservantur; cum synonymia critica et novarum specierum diagnosibus. Editio altera, auctior et emendatior. Uslar, Pyrmonti.

Merritt TJS, Shi L, Chase MC, Rex MA, Etter RJ, Quattro JM. 1998. Universal cytochrome b primers facilitate intraspecific studies in molluscan taxa. Molecular Marine Biology and Biotechnology 7: 7-11.

Meusnier I, Singer GAC, Landry JF, Hickey DA, Hebert PDN, Hajibabaei M. 2008. A universal DNA mini-barcode for biodiversity analysis. BMC Genomics 9: 214.

Michaud ALG. 1829. Description de plusieurs espèces nouvelles de coquilles vivantes. Bulletin d'Histoire Naturelle de la Société Linnéenne de Bordeaux 3: 260-276.

Michaud ALG. 1831. Complément de l'histoire naturelle des mollusques terrestres et fluviatiles de la France, de J.P.R. Draparnaud. Lippmann, Verdun.

Miller MA, Pfeiffer W, Schwartz T. 2010. Creating the CIPRES Science Gateway for inference of large phylogenetic trees. Proceedings of the Gateway Computing Environments Workshop (GCE), 14 Nov. 2010, New Orleans, LA pp 1-8.

Mörch OAL. 1864. Fortegnelse over de i Danmark forekommende Land- og Ferskvandsblöddyr. Videnskabelige Meddelelser fra den Naturhistoriske Forening i Kjöbenhavn 1863: 265367.

Nordsieck H. 2014. Annotated check-list of the genera of fossil land snails (Gastropoda: Stylommatophora) of western and central Europe (Cretaceous - Pliocene), with description of new taxa. Archiv für Molluskenkunde 142: 153-185.

Nylander JAA. 2004. MrModeltest v2. Evolutionary Biology Centre, Uppsala University. Program distributed by the author.

Palumbi S, Martin A, Romano S, McMillan WO, Stine L, Grabowski G. 1991. The simple fools guide to PCR. Special Publication of the Department of Zoology, University of Hawaii, Honolulu. 
Pfeiffer C. 1828. Naturgeschichte deutscher Land- und Süsswasser-Mollusken. Dritte Abtheilung. pp. I-VI [= 1-6], 1-84, Taf. I-VIII [= 1-8]. Weimar. (Landes-Industrie-Comptoir).

Pfeiffer KL. 1951. Chilostoma (Cingulifera) cingulata (Studer). Versuch einer monographischen Darstellung des Rassenkreises. Archiv für Molluskenkunde 80 (4/6): 89-214.

Pfeiffer L. 1848. Monographia heliceorum viventium. Sistens descriptiones systematicas et criticas omnium huius familiae generum et specierum hodie cognitarum 1: 1-484. Brockhaus, Lipsiae.

Pfeiffer, L. 1856. Bericht über weitere Mittheilungen des Herrn Zelebor. Malakozoologische Blätter 3: 175-186.

Pfeiffer L. 1868. Diagnosen neuer Heliceen. Malakozoologische Blätter 15: 83-85.

Rambaut A, Drummond AJ. 2007. Tracer v.1.5.0. Available from http://beast.bio.ed.ac.uk/Tracer

Ronquist F, Huelsenbeck JP. 2003. MrBayes 3: Bayesian phylogenetic inference under mixed models. Bioinformatics 19: 1572-1574.

Rooney AP, Piontkivska H, Masatoshi N. 2002. Molecular evolution of the nontandemly repeated genes of the histone 3 multigene family. Molecular biology and evolution 19: 68-75.

Rossmässler EA. 1834. Diagnoses conchyliorum terrestrium et fluviatilium. Verzeichniss zu Fascikeln natürlicher Exemplare 2 (21-40): 1-8.

Rossmässler EA. 1835-1837. Iconographie der Land- und Süßwasser-Mollusken, mit vorzüglicher Berücksichtigung der europäischen noch nicht abgebildeten Arten 1, 1-2: i-vi, 1-132, 2 pp., 1-26 [1835]; 3-4: 3 pp., 1-33, 3 pp., 1-27 [1836]; 5-6: 3 pp., 1-70 [1837]. Arnold, Dresden und Leipzig.

Rossmässler EA. 1838. Iconographie der Land- und SüßwasserMollusken, mit vorzüglicher Berücksichtigung der europäischen noch nicht abgebildeten Arten 2, 1-2: 1-43. Arnold, Dresden und Leipzig.

Roth JR. 1855. Spicilegium molluscorum terris orientalis provinciae mediterranensis peculiarium, ex novis inde reportatis collectionibus compilatum. 41 pp. Cassellis.

Schileyko AA. 1971. A comparative anatomical study of some Ariantinae (Pulmonata, Helicidae). Zoologicheskij Zhurnal 50: 990-1001. [In Russian]

Schileyko AA. 2006. Treatise on Recent terrestrial pulmonate molluscs. Part 13. Helicidae, Pleurodontidae, Polygyridae, Ammonitellidae, Oreohelicidae, Thysanophoridae. Ruthenica, Suppl. 2: 1765-1906.

Schileyko AA. 2013. Family Helicidae excluding Helicinae (Gastropoda Pulmonata): morphology, taxonomy, and a catalogue of taxa. Ruthenica 23: 127-162.

Schröter JS. 1784. Einleitung in die Conchylienkenntniß nach Linné II. Gebauer, Halle.

Stabile J. 1864. Mollusques terrestres vivants du Piémont. Atti della Società Italiana di Scienze Naturali 7(1): 3-141.

Strobel P. 1844. Delle conchiglie terrestri dei dintorni di Innsbruck. Giornale dell'Imperiale Reale Istituto Lombardo di Scienze, Lettere e Arti 9 (26): 301-331.

Strobel P. 1851. Notizie malacostatiche sul Trentino. Fusi, Pavia.

Strobel P. 1857. Essai d'une distribution orographico-géographique des mollusques terrestres dans la Lombardie. Memorie della Reale Accademia delle Scienze di Torino (2)18 ([1859]): 233-280 [= 1-50].

Studer R. 1820. Kurzes Verzeichniss der bis jetzt in unserm Vaterlande entdeckten Conchylien. Naturwissenschaftlicher
Anzeiger der Allgemeinen Schweizerischen Gesellschaft für die Gesammten Naturwissenschaften 3: 83-90, 91-94. Bern.

Sturany R. 1901. Diagnosen neuer Landschnecken aus der Hercegovina. Annalen des kaiserlich-königlichen Naturhistorischen Hofmuseums 16: 65-67. Wien.

Sturany R. 1907. Kurze Beschreibungen neuer Gastropoden aus der Merdita (Nordalbanien). Anzeiger der kaiserlichen Akademie der Wissenschaften, mathematisch-naturwissenschaftliche Klasse 44: 229-234.

Sturany R, Wagner AJ. 1914. Über schalentragende landmollusken aus Albanien und Nachbargebieten. Denkschriften der Mathematisch-Naturwissenschaftlichen Klasse der Kaiserlichen Akademie der Wissenschaften 91: 1-120.

Subai P. 1984. Über Bestrebungen zur Aufteilung der Subfamilie Ariantinae (= Helicigoninae, Campylaeinae). Mitteilungen der Deutschen Malakozoologischen Gesellschaft 37: 229-231.

Subai P. 1990. Beiträge zur Molluskenfauna des Epirus, I. Zwei neue Chilostoma-Arten aus Nord-Epirus (Gastropoda: Ariantinae). Annalen des Naturhistorischen Museums in Wien 91B: 243-247.

Subai P. 1995. Beitrag zur Kenntnis albanischer und griechischer Ariantinae (Gastropoda: Helicidae). Annalen des $\mathrm{Na}$ turhistorischen Museums in Wien 97 B: 75-94.

Subai P. 1996. Revision der Ariantinae. 1. Die Helicigona-Untergattung Thiessea (Gastropoda: Pulmonata: Helicidae). Archiv für Molluskenkunde 126: 1-49.

Subai P. 2002. Revision der Ariantinae. 2. Die Helicigona-Untergattung Liburnica (Gastropoda: Pulmonata: Helicidae). Archiv für Molluskenkunde 131: 1-65.

Subai P. 2012. Ergänzungen zur Revision der Ariantinae. Schriften zur Malakozoologie 27: 35-46.

Subai P, Fehér Z. 2006. Revision of the Ariantinae, 3. Superba n. gen., with the description of three new species. Archiv für Molluskenkunde 135: 205-223.

Sukumaran J, Holder MT. 2010. SumTrees - phylogenetic tree split support summarization Version 3.3.1. Part of the DendroPy Phylogenetic Computation Library. Bioinformatics 26: 1569-1571.

Swofford DL. 2002. PAUP*. Phylogenetic Analysis Using Parsimony (* and other methods). Version 4.0b10. Sinauer Associates, Sunderland, Massachusetts.

Thiesse J. 1884. Nouvelle hélice de Thessalie. Bulletins de la Société Malacologique de France 1: 271-272.

Tomić V. 1959. Sbirka retsentikh puzheva P.S. Pavlovića, u Prirodnjatshkom Muzeju u Beogradu. - P.S. Pavlovic's collection of the recent gastropods in the National History Museum in Beograd. Pozebna Izdanja, Prirodnjatshku Muzej u Beogradu 27: 1-74.

Turner H, Kuiper JGJ, Thew N, Bernasconi R, Ruëtschi M, Wüthrich M, Gosteli M. 1998. Atlas der Mollusken der Schweiz und Liechtensteins. Fauna Helvetica 2: 1-527.

Turton W. 1831. A manual of the land and freshwater shells of the British Islands arranged according to the more modern systems of classification; and described from perfect specimens in the author's cabinet: with coloured plates of every species. Longman, Rees, Irme, Brown and Greene, London.

Wagner A. 1928. Studien zur Molluskenfauna der Balkanhalbinsel mit besonderer Berücksichtigung Bulgariens und Thraziens, nebst monographischer Bearbeitung einzelner 
Gruppen. Annales Zoologici Musei Polonici Historiae Naturalis 6: 263-399.

Welter-Schultes FW. 2012. European non-marine molluscs: a guide for species identification. Planet Poster Ed.

Yule GU. 1924. A Mathematical Theory of Evolution, based on the Conclusions of Dr. J.C. Willis, F.R.S. Philosophical transactions of the Royal Society of London B, Biological sciences 213: 21-87.

Zilch A. 1960. Euthyneura. Handbuch der Paläozoologie 6, 2: 401-835.

Received: 6 April 2014

Revised and accepted: 28 August 2015

Published online: 28 January 2016

Editor: J.W. Arntzen

\section{Online Supplementary Information}

S1. MrBayes phylogeny based on the $H 3$ dataset S2. MrBayes phylogeny based on the COI dataset $S 3$. MrBayes phylogeny based on the $C y t B$ dataset S4. MrBayes phylogeny based on the $16 S$ dataset S5. BEAST phylogeny for the stringent $\mathrm{H3}-\mathrm{COI}-\mathrm{CytB}$ dataset S6. MrBayes phylogeny based on the relaxed $\mathrm{H3}-\mathrm{COI}-\mathrm{CytB}-16 \mathrm{~S}$ dataset S7. Taxa and sampling information 


\section{Appendix}

\section{Systematics}

Most nominal genus-group taxa, viz. Cattania, Corneola, Delphinatia, Dinarica, Faustina, Josephinella, Kosicia, Liburnica and Thiessea, are shown as distinct clades in our molecular analyses. These taxa are closely related to neither Campylaea nor Chilostoma and should no longer be regarded as subgenera of one of these genera. We consider the Ariantinae a subfamily with 21 genera (Table 5), five of which are subdivided into two (Campylaea, Dinarica, Liburnica), three (Chilostoma) or four (Cattania) subgenera. To some extent, the genetic distances may be used as a measure for the amount of (dis)similarity between taxa. For the status of taxa in the taxonomic hierarchy we use cladistic arguments, i.e. using identical ranking for clades that are sister-groups. Based on the phylogeny reconstructions we recognize the following genera and subgenera, listed in alphabetical order.

Subgenus nov. Achatica (monotypic), genus

\section{Chilostoma}

Type species: Helix achates Rossmässler, 1835

Abbreviations. PS = P. Subai; $\mathrm{RMNH}=$ Naturalis Biodiversity Center, Leiden; SMF = Forschungsinstitut Senckenberg, Frankfurt am Main

Diagnosis. The diagnosis of this monotypic subgenus is by definition identical with that of its type species, i.e. Chilostoma (Achatica) achates (Rossmässler, 1835). Achatica subgen. nov. is also differentiated by a unique combination of nucleotides in the 655 bp COI sequence obtained with general barcoding primers (Folmer, 1994) at the following relative positions: 79 C, 88 C, 181 C, 197 A, 211 C, 272 G, 319 C, 352 A, 538 G, 595 C.

Description. Shell strongly depressed, nearly discoid, rather dark, yellowish brown, with a brown spiral band; umbilicus wide (Kerney and Cameron, 1979: 201, pl. 21 fig. 2; Boschi, 2011: 570-571; Welter-Schultes, 2012: 580). See "Chilostoma (Chilostoma) achates" in Schileyko (2006: fig. 2264B, C; 2013: 143, fig. 13) for details regarding the genital morphology. The accessory glands are undivided.

Molecular data. Two individuals have been used for the molecular analyses, viz. (a) a specimen collected in the northern limestone Alps (Berchtesgaden, Bayern, Germany), and (b) a specimen from the south- ern limestone Alps (Greifenburg, Kärnten, Austria). The sequence divergences between these specimens, based on all four markers, is less than $0.2 \%$ (Table 4). When compared to sequence divergences of 3.3-6.5\% between subspecies of Chilostoma (Cingulifera), there is at least no genetic support for a classification of these two populations of C. (Chilostoma) achates as different subspecies (as suggested by Falkner, 1998).

Age. The unresolved sister-group relationships between the subgenera of Chilostoma (see Chilostoma [Chilostoma]) do not allow for an unequivocal estimate for the emergence of Chilostoma (Achatica). If it dates back to the most basal node within the genus (Fig. 2), it is estimated at ca. 38.5 MYA (Fig. 2). When C. (Achatica) and C. (Cingulifera) are sister-groups (Fig. S5) the most recent common ancestor is estimated at ca. 24.7 MYA (Fig. S5).

Distribution. Austria, E Switzerland, S Germany (Bayern, Berchtesgadener Alps), N Italy.

Remarks. It is surprising that only a single, polytypic species is classified in Achatica, because Chilostoma (C.) adelozona (Strobel, 1857) and Chilostoma (C.) zonatum (Studer, 1820) have brown shells that look similar to $C$. (A.) achates at first sight. See Chilostoma (Chilostoma).

Derivatio nominis. The name Achatica is supposed to recall the name of the type species.

Genus Arianta Turton, 1831

Type species: Helix arbustorum Linné, 1758

Molecular data. Four Arianta species could be studied, viz. A. aethyops (Bielz, 1851), A. arbustorum s. lat. (with five subspecies, two of which are considered separate species by some authors [Welter-Schultes, 2012]), A. chamaeleon (Pfeiffer, 1868), and A. schmidtii (Rossmässler, 1836). The monophyly of this broadly accepted genus is supported in all molecular phylogeny reconstructions (PP $=1.0$; Figs 1,2 , S1-S6). The position of $A$. arbustorum stenzii (see Gittenberger et al. 2004) or A. arbustorum stenzii-arbustorum in phylogeny reconstructions presented in this study does not give any support for the introduction of Altarianta Schileyko, 2013, as a subgenus of Arianta. This is in accordance with the fact that $A$. a. stenzii and $A$. $a$. arbustorum, hybridize where they are in contact. Arianta chamaeleon, which is shown as the sister-group of the other Arianta species, is less closely related. The 
conchologically unexpected sister-group relationship ( $\mathrm{PP}=1.0$; Figs 1-2, S1, S3-S6) between Arianta and Cylindrus (Fig. 3.3) was shown by Groenenberg et al. (2012) and has recently been confirmed by Cadahia et al. (2013).

Age. The most recent common ancestor for Arianta is estimated at ca. 23.1-22.4 MYA (Figs. S5, 2).

Distribution. Arianta arbustorum (Fig. 3.1) has the largest distribution range of all the species within the subfamily Ariantinae. It occurs in north and central Europe, from Iceland, Norway, Sweden, N.-Ireland, Great Britain, and central France eastwards to the Baltic countries, Poland, Ukraine and Romania (Carpathians). The southern border ranges from the N.-Italian Alps through Slovenia, Croatia and Serbia into Bulgaria (up to Stara Planina); except for some localities

Fig 3. Compilation of shells representing most of the currently recognized genera of Ariantinae. 1. Arianta arbustorum (Linnaeus, 1758) B $24.1 \mathrm{~mm}$ [RMNH G2131] Austria, Steiermark, near Gstatterboden; E. Gittenberger leg., 10-IX-1964. 2. Arianta chamaeleon wiedermayeri (Kobelt, 1903) B 18.3 mm [RMNH G2608] Austria, East Tirol, S of Kartitsch; E. Gittenberger leg., VIII-1974. 3. Cylindrus obtusus (Draparnaud, 1805) B $13.7 \mathrm{~mm}$ [RMNH 73877] Austria, Oberösterreich, Bledigupf; W.H. Neuteboom leg., 22-VII1966. 4. Isognomostoma isognomostomos (Schröter, 1784) B 9.7 mm [RMNH 74226] Austria, Kärnten, Plöckenpass; W.H. Neuteboom leg., 14-IX-1952. 5. Causa holosericea (Studer, 1820) B 10.5 mm [RMNH 74311] Austria, Salzburg, Amerthal; W.H. Neuteboom leg., 16-VII-1968. 6. Chilostoma (Achatica) achates (Rossmässler, 1835) B 21.9 mm [RMNH G2410] Austria, Steiermark, E of Brandtriedl; A. and E. Gittenberger leg., 19-V-1972. 7. Chilostoma (Chilostoma) zonatum rhaeticum (Strobel, 1857) B 25.2 [RMNH G54412] Switzerland, Graubünden, E of Martinsbruck; E. Gittenberger leg., IX-1963. 8. Chilostoma (Chilostoma) tigrinum (De Cristofori and Jan, 1832) B $24.7 \mathrm{~mm}$ [RMNH 73434] Italy, Como, Pasturo; W.H. Neuteboom leg., 3-VIII-1954. 9. Chilostoma (Cingulifera) cingulatum cingulatum (Studer, 1820) B $20.5 \mathrm{~mm}$ [RMNH H1938] Switzerland, Tessin, Melide along Lago di Lugano; J.T. Henrard leg., 28.VIII.1938. 10. Chilostoma (Cingulifera) cingulatum gobanzi (Frauenfeld, 1867) B $22.8 \mathrm{~mm}$ [RMNH 73408] Italy, Brescia, Val Toscolano; W.H. Neuteboom leg., 05-VIII-1954. 11. Delphinatia fontenillii alpina (Michaud, 1831) B 19.6 mm

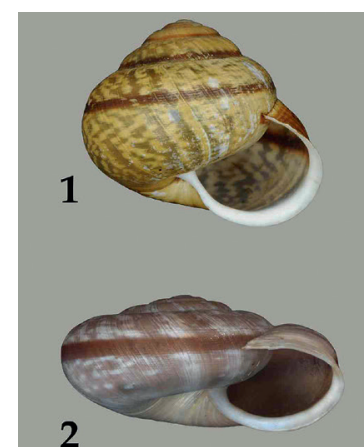
2

3

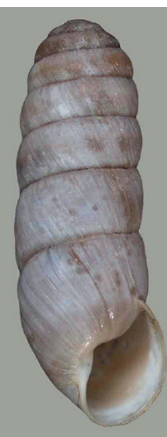

5
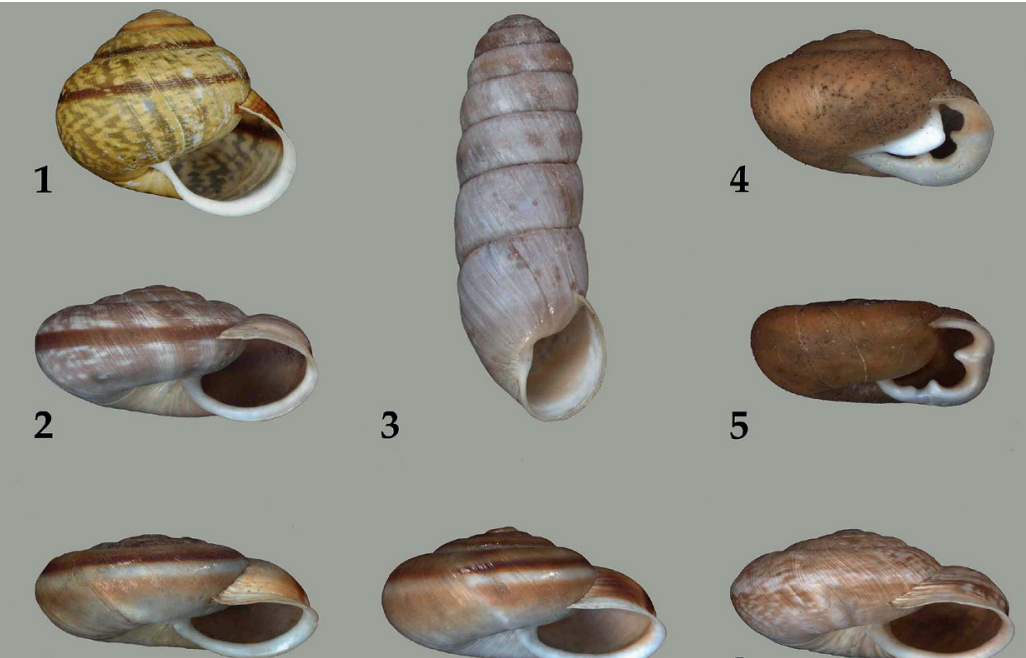

6

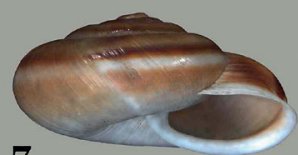

7
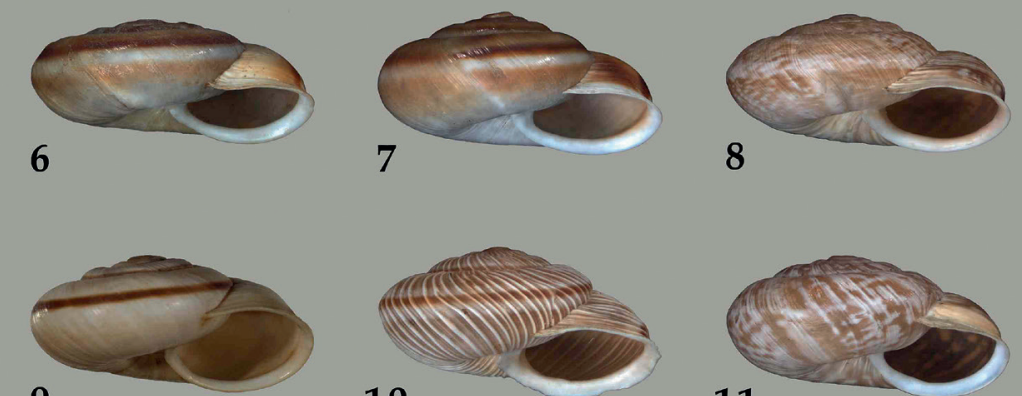

9

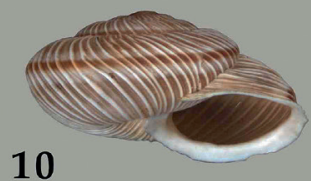

10
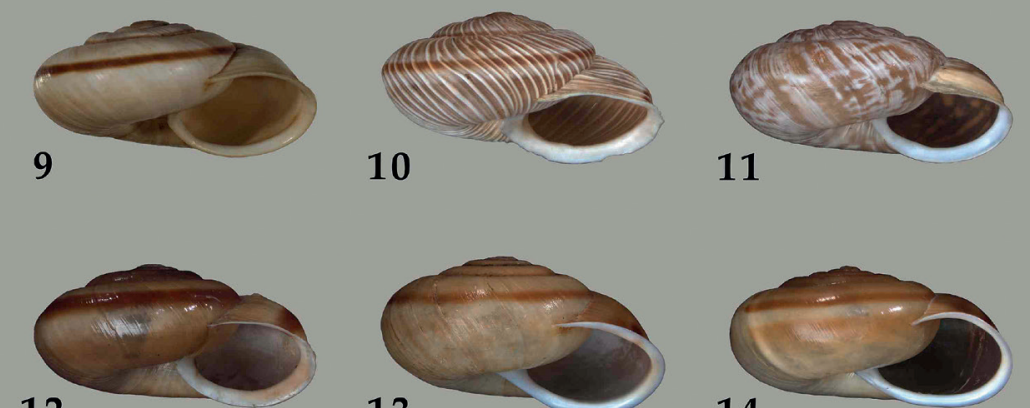

12

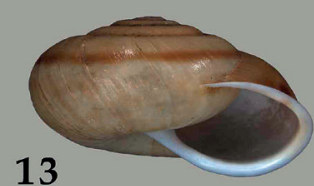

14
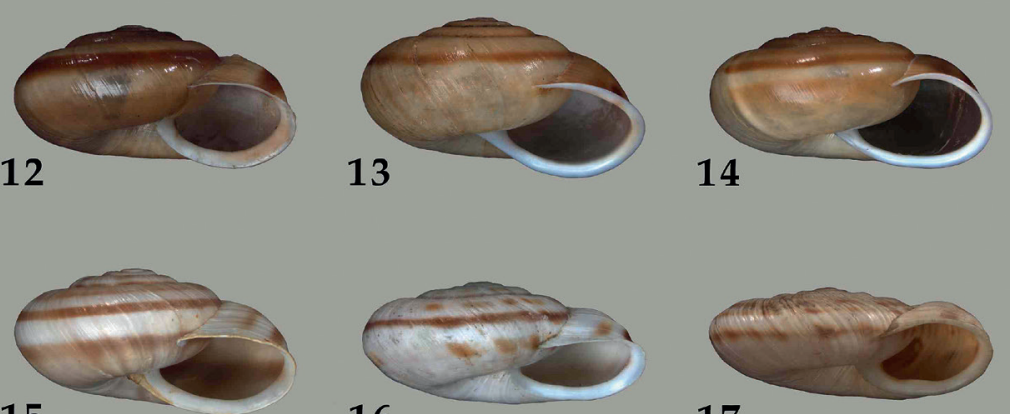

15

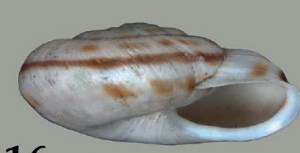

16

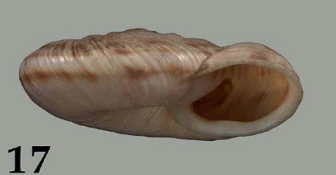

[RMNH G3646] France, Isère, SSE of Laurent-du-Pont; E. Gittenberger leg., 12-IX-1975. 12. Faustina faustina (Rossmässler, 1835) B 19.6 mm [RMNH 53576] Hungary, Bükk, Szalajkavölgy; Agócsy leg., 21-V-1921. 13. Campylaea (Campylaea) planospira planospira (Lamarck, 1822) B 25.9 mm [RMNH 73625] Italy, Torino, Santvaris di Montebruno; W.H. Neuteboom leg., 8-VII-1977. 14. Campylaea (Oricampylaea) illyrica (Stabile, 1864) B 25.4 mm [RMNH 11124] Italy, Friuli, SSE of Tarvisio; E. Gittenberger leg., VI-1992. 15. Kosicia ambrosi (Strobel, 1852) B 12.5 mm [RMNH 24940] Italy, Vicenza, Valstagna; W.H. Neuteboom leg., 22-VII-1968. 16. Kosicia intermedia (Pfeiffer, 1828) B 15.3 mm [RMNH 54440] Austria, Kärnten, Deutschpeter; E. Gittenberger leg., IX-1964. 17. Kosicia ziegleri (Rossmässler, 1836) B 17.7 mm [RMNH G2350] Slovenia, Kamniške Alps, Igla Studenec; A. and E. Gittenberger leg., 26-VIII-1971. 
in the Spanish Pyrenees south of the watershed (A. arbustorum xatarti Farines, 1834) it does not occur in the Iberian peninsula.

Remarks. Four or five Arianta species have been described, some of which are polytypic. Campylaea apfelbecki Sturany, 1901, which was considered a subspecies of A. chamaeleon by Knipper (1939), could not be investigated; it might be either a fifth Arianta spe- cies or belong to Cattania (Cattaniella). The exceptionally widespread Arianta arbustorum arbustorum is aberrant also in terms of shell morphology and in its ecological requirements, occurring independently of limestone from the lowland to high in the mountains. The other Arianta species are restricted to (high) alpine habitats. While nearly all Ariantinae have a depressed shell and an open umbilicus, A. a. arbustorum

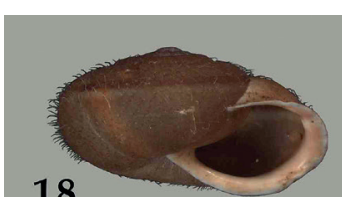

18
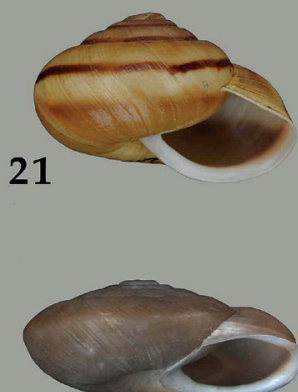

24
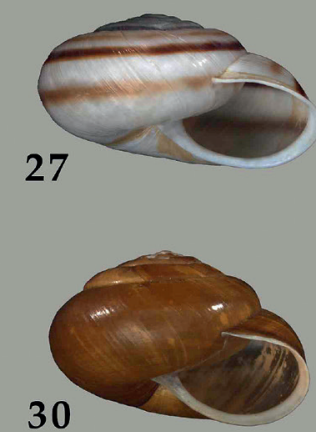

30

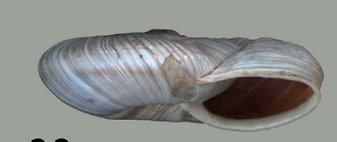

33

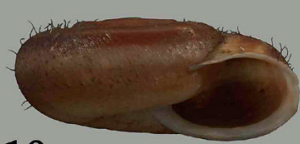

19
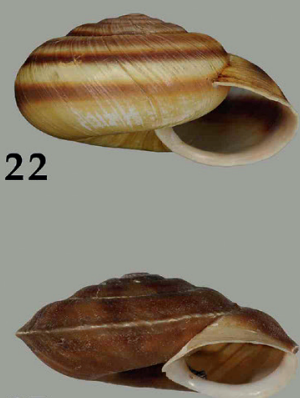

25
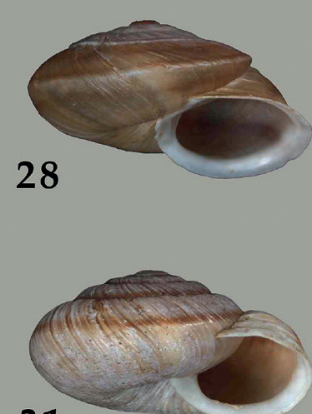

31

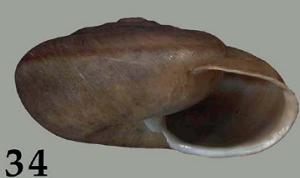

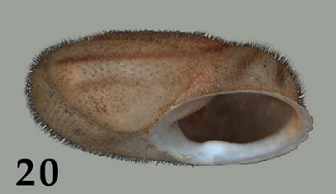

20
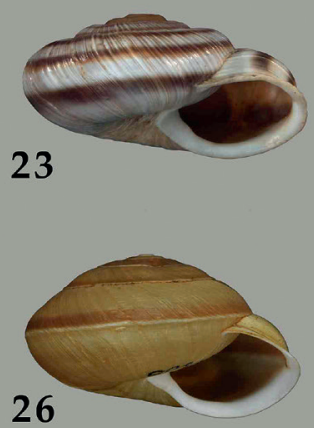

29
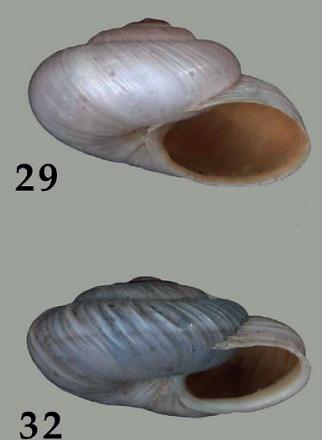

32

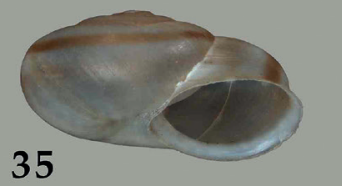

18. Campylaeopsis moellendorffii (Kobelt, 1871) B $18.4 \mathrm{~mm}$ [RMNH AJW898] Bosnia-Herzegovina, Vrelo Bosne, near Ilidza; A.J. de Winter leg., 17-IX-1980. 19. Kollarix kollari (Pfeiffer, 1856) B 24.3 mm [RMNH YU.429] Serbia, along Ovcar Banja; W.J.M. Maassen leg., V-1984. 20. Liburnica (Liburnica) setosa setosa (Férussac, 1832) B $23.6 \mathrm{~mm}$ [RMNH 94272] Croatia, Lovrec, S of Imotski; W.J.M. Maassen leg., IV-1989. 21. Dinarica (Sabljaria) stenomphala (Menke, 1830) B 30.7 mm [col. PS.21228] Croatia, Velebit Mts, near Krasno Polje; P. Subai leg., 29-VII2002. 22. Dinarica (Dinarica) pouzolzii (Deshayes, 1830) B $40.1 \mathrm{~mm}$ [RMNH 53508 / 413] Croatia, Dalmatia, E of Biokovo Mts; J.J. ter Pelkwijk leg., 12-VIII1939. 23. Liburnica (Superba) skipetarica skipetarica (Subai, 1995) B 19.6 mm [col. PS.20215] Albania, Periferi Berat, Tommorit; P. Subai leg., VIII-2004. 24. Corneola desmoulinsii (Farines, 1834) B 17.2 mm [RMNH 93925] Andorra, Canillo, northern wall; W.J.M. Maassen leg., VII1990. 25. Helicigona lapicida lapicida (Linnaeus, 1758) B 17.6 mm [SMF3254 26/1] Germany, Hessen, Schlüchtern; M. Pfenninger leg. 26. Drobacia banatica (Rossmässler, 1838) B 29.5 mm [RMNH 54500 / 485] Romania, Siebenbürgen; H. de Wever leg. 27. Cattania (Cattania) trizona (Rossmässler, 1834) B $24.4 \mathrm{~mm}$ [RMNH 99615] Romania, Banat Mts, Mt Domogled; Kroupa leg., 21-VI-1985. 28. Cattania (Cattania) subaii (Fauer, 1991) B 22.9 mm [RMNH GU.9921 / EK5558] Greece, Makedonia, W of Kozani; E. Gittenberger and D. Uit de Weerd leg., 23-V1999. 29. Vidovicia caerulans (Pfeiffer, 1828) B 15.4 mm [RMNH 93836] Croatia, Velebit near Starigrad; W.J.M. Maassen leg., IX-1982. 30. Cattania (Ariantopsis) pelia (Hesse, 1912) B 17.9 mm [col. PS.23572] Bulgaria, Vitosha, Bistrisko branishte; I. Dedoy leg., 8-VII-2004. 31. Cattania (Wladislawia) polinskii (Wagner, 1928) B 16.4 mm [RMNH G3749] Bulgaria, Pirin Mts, Mt Vihren; A. Riedel leg., 24-VI-1977. 32. Cattania (Wladislawia) sztolcmani (Wagner, 1928) B 10.6 mm [RMNH G3749] Bulgaria, Pirin Mts, Mt Vihren; A. Riedel leg., 24-VI-1977. 33. Josephinella vikosensis (Subai, 1990) B 18.8 mm [RMNH EG.9703 / DK8112] Greece, Ipiros, Vikos valley; E. Gittenberger leg., 23-VII-1997. 34. Josephinella hemonica (Thiesse, 1884) B $19.1 \mathrm{~mm}$ Greece, Makedhonia, SE of Grevena; E. Gittenberger leg., 18-VII-1986. 35. Thiessea sphaeriostoma (Bourguignat, 1857) B 21.0 mm [RMNH 75078] Greece, Sterea Ellas, SE of Mariolates; E. Gittenberger and D. Uit de Weerd leg., 19-V-2000. 
has a globular shell with a closed umbilicus (Gittenberger et al., 2004). Other Arianta species, such as A. chamaeleon (Fig. 3.2) and A. schmidtii retained the plesiomorphic, depressed shell phenotype. Some subspecies of A. arbustorum that are characterized by depressed shells (Gittenberger et al., 2004; Haase and Misof, 2009) might have evolved that character state by reversal. See also Arianta in Schileyko (2013) for details regarding the genital morphology. The accessory glands are undivided (Fig. 4.1). Specimens of $A$. arbustorum stenzii from several localities should be dissected to investigate the status of Altarianta Schileyko, 2013, in more detail.

Subgenus Ariantopsis Wagner, 1928 (monotypic), genus Cattania

Type species: Helicigona (Arianta) pelia Hesse, 1912

Molecular data. Both MrBayes and BEAST phylogeny reconstructions for the relaxed dataset highly support a sister-group relation $(\mathrm{PP}=1.0)$ between Ariantopsis and Wladislawia (Figs 2, S6), which are here considered subgenera of Cattania Brusina, 1904.

Age. The most recent common ancestor of Cattania (Ariantopsis) and C. (Wladislawia) is estimated at ca. 7.3 MYA (Fig. 2).

Distribution. Ariantopsis is endemic to SW and W Bulgaria. The eastern boundary of its distribution is situated near Plovdiv, the northern boundary is near Lakatnik in the Iskar-valley, and its western distribution is formed by Mt Vitosha and the Rila Mts.

Remarks. The taxonomic position of Cattania (Ariantopsis) pelia (Fig. 3.30) has long been uncertain. Conchologically it somewhat resembles Arianta aethyops. It has been assigned to various genera, viz. Arianta by Kroupa (1994) and Dedov (1998), Helicigona by Hesse (1912), Chilostoma by Bank et al. (2001) and Faustina by Damjanov and Likharev (1975). See also Campylaea (Ariantopsis) and Ariantopsis in Schileyko (2006, 2013) for details regarding the shell and genital morphology. The accessory glands can be undivided, but are mostly split up to half of their length (Fig. 4.29).

Subgenus Campylaea Beck, 1837 (monotypic?), genus Campylaea

Type species: Campylaea planospira Lamarck, 1822

Molecular data. Campylaea (C.) planospira (Fig. 3.13 ) is the sister-group of a clade with three species, referred to below as Campylaea (Oricampylaea) (PP $>0.92$; Figs S1, S2). Together, the subgenera Campylaea and Oricampylaea, form a monophyletic group ( $\mathrm{PP}=1.0$; Figs 2, S1, S2, S4, S6), viz. the genus Campylaea. The genetic distances between $C$. (Campylaea) and $C$. (Oricampylaea) are comparatively large (COI sequence divergence up to $22.1 \%$; Table 4). It is unclear to which genus Campylaea is most closely related. Except for the phylogeny reconstruction according to $\mathrm{COI}$, which suggest a sister-group relationship between Campylaea and Kollarix (PP = 0.86; Fig. S2), none of the other datasets provides information regarding possible sister-group relationships of Campylaea. In the phylogenies based on the concatenated datasets Campylaea branches off early in either group A (Figs 1, S6) or group B (Fig. 2).

Age. The most recent common ancestor of Campylaea is estimated at ca. 34.0 MYA (Fig. 2).

Distribution. Campylaea (C.) planospira is represented in S Austria, N Balkans, mainland Italy, and the island of Sicily.

Remarks. See also Campylaea (Campylaea) and Chilostoma (Campylaea) in Schileyko (2006, 2013) for details regarding the shell and genital morphology. The accessory glands can be undivided (Fig. 4.14), but Sturany and Wagner (1914) and Knipper (1939) showed split accessory glands in C. (Campylaea) planospira. Penial papilla small, conical, with fine transverse ridges and an obtuse apex with a short, transverse, slit-like pore. Secondary ureter entirely open. For the moment being, only a single, polytypic species is accepted in Campylaea s. str. Some of the so-called subspecies could be considered separate species, however.

Genus Campylaeopsis Sturany and Wagner, 1914 (monotypic)

Type species: Helicigona moellendorffii Kobelt, 1871.

Molecular data. Only an $H 3$ sequence was obtained for this taxon. In the respective phylogeny Campylaeopsis is placed in a clade with Delphinatia, Drobacia, Pseudotrizona, and Vidovicia ( $\mathrm{PP}=0.8$; Fig. S1). Campylaeopsis moellendorffii shares a substantial part of its distribution area with Pseudotrizona inflata.

Age. Not enough sequence information was obtained to include Campylaeopsis in the time calibrated analyses (Figs. 2, S5).

Distribution. The mountains of Bosnia-Herzegowina and Montenegro. 
Remarks. Campylaeopsis moellendorffii (Fig. 3.18) has a characteristic shell with regularly arranged, widely spaced hairs. It has been assigned to Helicigona by Knipper (1939) and to Chilostoma by Bank (2001).

See also Helicigona (Campylaeopsis) and Chilostoma (Campylaeopsis) in Schileyko (2006, 2013) for details regarding the shell and genital morphology. The accessory glands are undivided (Fig. 4.10).

Subgenus Cattania Brusina, 1904, genus Cattania Type species: Helix trizona Rossmässler, 1835

Molecular data. Five of the ca. 9 Cattania (C.) species are included in this study. The genus Cattania is shown to be monophyletic ( $\mathrm{PP}=1.0$; all pylogenies except S1). It includes the subgenera: Cattania (Ariantopsis), C. (Cattania), Cattania (Cattaniella) and C. (Wladislawia). Cattania is the sister-group of the clade Josephinella-Thiessea (PP $\geq 0.8$; Figs 1, 2, S1-S3 and S6). Cattania (Cattania) constitutes a monophyletic group within Cattania ( $\mathrm{PP}=1.0$; Fig. S2) and is in this study represented by $C$. (C.) faueri, C. (C.) kattingeri, $C$. (C.) pseudocingulata, $C$. (C.) subaii and the type species $C$. (C.) trizona. It is the sister-group of the clade C. (Ariantopsis)-C. (Wladislawia) ( $\mathrm{PP} \geq 0.95$; Figs S2, S6). The $C O I$ sequence divergences between $C$. (Cattania) and C. (Ariantopsis), and between the former and C. (Wladislawia) are $13.4 \%$ and $14.7 \%$, respectively. The data of Cadhaia et al. 2013 show that C. (Cattania) haberhaueri belongs to this subgenus as well. Future research will have to make clear whether C. balcanica and C. rumelica should also be classified here. All phylogeny reconstructions indicate that the species referred to as Cattania inflata (Kobelt, 1876) by Subai (1995) represents a separate lineage (Figs 1, 2, S1-S6) that is clearly distinct from Cattania. We consider this lineage a separate genus, referred to below as Pseudotrizona gen. nov.

Age. The most recent common ancestor of Cattania is estimated at ca. 27.6-26.9 MYA (Figs 2, S5).

Distribution. Central Balkans, SW Romania, E and S Serbia, SW Bulgaria and N Greece (Thraki).

Remarks. See Campylaea (Cattania) and Chilostoma (Cattania) in Schileyko $(2006,2013)$ for details

Table 4. Uncorrected p-distances (in percentages) for a selection of taxa.

\begin{tabular}{|c|c|c|c|c|c|}
\hline From & To & & $\mathrm{H} 3$ & $\mathrm{COI}$ & $\mathrm{CB}$ \\
\hline 25 Campylaea (Campylaea) planospira & 26 & Campylaea (Oricampylaea) illyrica & 2,4 & 22,1 & - \\
\hline 25 Campylaea (Campylaea) planospira & 30 & Campylaea (Oricampylaea) lefeburiana & 2,4 & 20,5 & - \\
\hline 41 Cattania (Cattania) subaii & 34 & Cattania (Ariantopsis) pelia & 0 & 13,4 & 18 \\
\hline 41 Cattania (Cattania) subaii & 46 & Cattania (Wladislawia) sztolcmani & 0,3 & 14,7 & - \\
\hline 41 Cattania (Cattania) subaii & 45 & Cattania (Cattaniella) thateensis & 0,3 & 15,4 & - \\
\hline 54 Causa holosericea & 125 & Isognomostoma isognomostomos & 1,2 & 20,2 & 23 \\
\hline 63 Chilostoma (Chilostoma) zonatum adelozona & 83 & Chilostoma (Cingulifera) cingulatum preslii & 0 & 14,5 & 18,6 \\
\hline 63 Chilostoma (Chilostoma) zonatum adelozona & 57 & Chilostoma (Achatica) achates & 0 & 14,7 & 18,6 \\
\hline 83 Chilostoma (Cingulifera) cingulatum preslii & 57 & Chilostoma (Achatica) achates & 0 & 14,8 & 18 \\
\hline 57 Chilostoma (Achatica) achates & 58 & Chilostoma (Achatica) achates & 0 & 0,2 & 0,3 \\
\hline 72 Chilostoma (Cingulifera) cingulatum peregrini & 74 & Chilostoma (Cingulifera) cingulatum preslii & 0,3 & 0,6 & 0,6 \\
\hline 72 Chilostoma (Cingulifera) cingulatum peregrini & 86 & Chilostoma (Cingulifera) cingulatum preslii & 0,3 & 0 & 0 \\
\hline 90 Corneola squamatinum & 88 & Corneola desmoulinsii & 0,6 & 16,9 & - \\
\hline 94 Cylindrus obtusus & 18 & Arianta chamaeleon & 0,9 & 18,8 & 24,9 \\
\hline 94 Cylindrus obtusus & 9 & Arianta arbustorum arbustorum & 0,9 & 20 & 23,8 \\
\hline 100 Dinarica (Dinarica) pouzolzii & 102 & Dinarica (Dinarica) serbica & 0,9 & 11 & 13,6 \\
\hline 100 Dinarica (Dinarica) pouzolzii & 103 & Dinarica (Sabljaria) stenomphala & 1,8 & 16 & 24,7 \\
\hline 110 Faustina faustina orba & 111 & Faustina kiralikoeica & 0 & 17,4 & - \\
\hline 107 Faustina faustina associata & 108 & Faustina faustina faustina & 0 & 10,7 & 13 \\
\hline 113 Helicigona lapicida andorrica & 115 & Helicigona lapicida andorrica & 0 & 0,3 & 0,3 \\
\hline 119 Helicigona lapicida lapicida & 117 & Helicigona lapicida lapicida & 0,6 & 1,8 & 0,6 \\
\hline 113 Helicigona lapicida andorrica & 117 & Helicigona lapicida lapicida & 0,6 & 11,8 & 14,1 \\
\hline 154 Liburnica (Liburnica) setosa & 149 & Liburnica (Liburnica) albanograeca & 0 & 3,4 & - \\
\hline 150 Liburnica (Liburnica) dunjana & 153 & Liburnica (Liburnica) setigera setigera & 0 & 6,3 & - \\
\hline 158 Liburnica (Superba) skipetarica & 156 & Liburnica (Superba) kulmankana & 0 & 2,1 & - \\
\hline 153 Liburnica (Liburnica) setigera setigera & 157 & Liburnica (Superba) skipetarica & 0 & 6,9 & - \\
\hline
\end{tabular}


regarding the shell and genital morphology. In $C$. (Cattania) the accessory glands are usually split (Fig. 4.27); occasionaly one of the glands is undivided.

Subgenus nov. Cattaniella, genus Cattania Type species: Helix cingulata olympica Roth, 1855

Diagnosis. The two Cattaniella species share a unique combination of nucleotides in the $655 \mathrm{bp} C O I$ sequence obtained with general barcoding primers (Folmer, 1994) at the following relative positions: $87 \mathrm{~A}, 95 \mathrm{~T}, 235$ A, 331 G, 365 G, 406 T, 499 G, 542 A, 543 G, 583 A.

Description. Shell depressed globular to low conical, nearly discoid, whitish, corneous or brown, with one to three brown spiral bands. The accessory glands in C. (Cattaniella) thateensis are split (Subai, 2012).

Molecular data. Cattania (Cattaniella) is represented by $C$. (Cattaniella) olympica and C. (Cattaniella) thateensis (Subai, 2012). It is the sister-group of the combined three other subgenera (Figs 2, S2, S3, S6) of Cattania. Clearly C. (Cattaniella) olympica should no longer be considered a subspecies of $C$. (Cattania) trizona (see Knipper, 1939). Likewise, $C$. (Cattaniella) thateensis cannot be classified in Wladislawia (see Subai, 2012). Future research will have to show whether Campylaea apfelbecki Sturany, 1901 and Campylaea zebiana Sturany, 1907 belong to Cattania (Cattaniella) as well (Subai, 2012).

Distribution. Higher montane areas of E Albania (Thäte mountains); Olympos and Ossa mountain areas of Thessaly, Greece.

Remarks. For the moment being, only the two species that could be investigated for this study are classified in Cattaniella.

Derivatio nominis. Cattaniella refers to Cattania.

Genus Causa Schileyko, 1971 (monotypic)

Type species: Glischrus (Helix) holosericea Studer, 1820

Molecular data. The sister-group relationship, as well as a substantial genetic distance between Causa and Isognomostoma are established ( $\mathrm{PP}=1.0$; Figs 1, 2, S2-S6; Table 4). Only the $H 3$ data failed to show a direct sister-group relation, but still placed both genera in the same clade ( $\mathrm{PP}=0.42$, Fig. $\mathrm{S} 1)$.

Age. The most recent common ancestor of Causa and Isognomostoma is estimated at ca. 33.0-30.1 MYA (Figs 2, S5).
Distribution. Alps, Sudetes and W Carpathians (Tatra Mts), isolated in S Germany (Franconian Jura).

Remarks. Conchologically, Causa holosericea (Fig. 3.5) and Isognomostoma isognomostomos (Fig. 3.4) are both aberrant among the Ariantinae by the dentate aperture. These species were considered congeneric until Schileyko (1971), primarily based on differences in genital anatomy, introduced Causa as a new genus. See also Causa in Schileyko (2006, 2013) for details regarding the shell and genital morphology. The accessory glands in both Causa and Isognomostoma are undivided (Fig. 4.7-4.8).

Subgenus Chilostoma Fitzinger, 1833, genus Chilostoma

Type species: Glischrus (Helix) foetens Studer, 1820

Molecular data. Four or five species can be classified in Chilostoma s. str., three of which are included in this study. Within the genus Chilostoma $(\mathrm{PP}=1.0$; Figs 1, 2, S2, S3, S5, S6), three well supported clades can be discerned: I) Chilostoma (Chilostoma), II) Chilostoma (Cingulifera) Held, 1838 and III) Chilostoma (Achatica) subgen. nov. The COI sequence divergences between each of the three subgenera are about $15 \%$. Chilostoma is the sister-group of all other Ariantinae taxa in group B ( $\mathrm{PP} \geq 0.6$, Figs 1,2 , S6). Only Figs S3 and S5 specifically indicate Corneola as its sister-group ( $\mathrm{PP}=0.94)$. The latter relationship is not observed if both of the studied Corneola species are included (Figs 2, S1, S2, S6). The phylogenetic relationships between the subgenera of Chilostoma are not resolved. Figs 2, S4 and S6 support (PP $\geq 0.95)$ a sistergroup relationship between $C$. (Chilostoma) and $C$. (Cingulifera), whereas Figs 1, S3 and S5 indicate Chilostoma (Achatica) as the sister-group of C. (Cingulifera) $(\mathrm{PP} \geq 0.95)$.

Age. The most recent common ancestor of the genus Chilostoma is estimated at ca. 38.5-31.3 MYA, whereas that of $C$. (Chilostoma) is estimated at ca. 20.3-18.4 MYA (Figs 3 and S5).

Distribution. The Alps (SE France, S Switzerland, N Italy).

Remarks. Unexpectedly, from a conchological perspective, C. (Chilostoma) zonatum (Fig. 3.7) turns out to be more closely related to $C$. (Chilostoma) frigidum and $C$. (Chilostoma) tigrinum (Fig. 3.8), than to $C$. (Achatica) achates (Fig. 3.6), which shares the chestnut brown colour of the shell. That colour might be the plesiomorphic character state in Chilostoma. Nowa- 
days, Helix foetens is either synonymised with $C$. (Chilostoma) zonatum, as by Turner et al. (1998) or it is considered a subspecies of that species (Bank et al., 2001). In the past many subgenera have been assigned to Chilostoma (Zilch, 1960; Bank et al., 2001). It is unclear which, if any, character states of the genital tract are diagnostic for the subgenera of Chilostoma. The accessory glands are undivided (Fig. 4.3, 4.4).

Subgenus Cingulifera Held, 1838 (monotypic), genus Chilostoma

Type species: Glischrus (Helix) cingulata Studer, 1820. The type species can be subdivided into several subspecies, five of which are included in this study

Molecular data. See Chilostoma (Chilostoma).

Age. The most recent common ancestor of C. (Cingulifera) is estimated at ca. 7.9-7.0 MYA (Figs 2, S5). The split between $C$. (Cingulifera) and either $C$. (Chilostoma) or $C$. (Achatica) subgen. nov. (see Chilostoma) is estimated at ca. 29 and 24.7 MYA, respectively.

Distribution. NE Italy, SW Austria, SE Switzerland, locally in the French Alps, Central Italy and S Germany (partly introduced). Chilostoma (Chilosto$m a$ ) and $C$. (Cingulifera) have a parapatric distribution. Generally the former subgenus is distributed in the western Alps, whereas the latter one has its main range in the eastern Alps. Additionally, our preliminary results indicate a strong separation between the Chilostoma species east versus west of the Camonica valley (Valcamonica, Italy).

Remarks. Chilostoma (Cingulifera) is a generally accepted subgenus of Chilostoma (Zilch, 1960; Bank et al., 2001). Taxonomically it was supposed to encompass only a single species, i.e. Chilostoma (Cingulifera) cingulatum (Studer, 1820) (Fig. 3.9, 3.10) with a large number of alleged subspecies (Pfeiffer, 1951), some of which are here classified differently, however, viz. Chilostoma (C.). frigidum and Chilostoma (C.) tigrinum (De Cristofori and Jan, 1832; Fig. 3.8).

Chilostoma (Cingulifera) cingulatum peregrini Falkner, 1998 was introduced as a replacement name for Chilostoma (Cingulifera) cingulatum cingulina (Strobel, 1844), not Helix cingulina Deshayes, 1839 (in Férussac and Deshayes). Contrary to the prevailing view, Falkner suggested that the northern alpine populations of Chilostoma (Cingulifera) cingulatum might belong to two instead of only a single subspecies, viz. Chilostoma (Cingulifera) c. peregrini from near Inns- bruck (Austria) and Chilostoma (Cingulifera) c. preslii from near Berchtesgaden (Falkner, 1998; KierdorfTraut, 2012). $C O I$ and $C y t B$ sequences for specimens from both northern alpine localities (Table S7, 72-73 versus 83-86) are virtually identical and differ about $0.5 \%$ (Table 4) from sequences of southern alpine, undisputed Chilostoma (Cingulifera) c. preslii (78-82, Table S7). These data indicate that Chilostoma (Cingulifera) c. peregrini is a junior synonym of Chilostoma (Cingulifera ) c. preslii, which has a disjunct range, occurring in both the northern and the southern limestone Alps. See Helicigona (Cingulifera) and Chilostoma (Cingulifera) in Schileyko $(2006,2013)$ for details regarding the shell and genital morphology. Chilostoma (Cingulifera) has undivided accessory glands (Fig. 4.4).

Genus Corneola Held, 1838

Type species: Helix cornea Draparnaud, 1801

Molecular data. Corneola squamatinum (Rossmässler, 1835) and C. desmoulinsii (Farines, 1834) together are monophyletic $(\mathrm{PP}=0.86$ and 0.77 ; Figs $2, \mathrm{~S} 6)$. The COI sequence divergence between these species is $16.9 \%$ (Table 4). The phylogeny reconstructions are indistinct regarding the position of this genus. In the concatenated analyses it is shown between Campylaea and Chilostoma (Fig 1), as the sister-group of either of these (Figs S6 and S5) or as the sister-group of Causa, Isognomostoma and Helicigona (Fig. 2). Corneola is here regarded as a genus.

Age. The most recent common ancestor of Corneola is estimated at ca. 52.5 MYA (Fig. 2).

Distribution. Corneola acrotricha (Fischer, 1877) and $C$. desmoulinsii are mainly found in the Pyrenees. Corneola squamatinum extends also further into southern and central France, along the Atlantic coast up to Brittany, whereas C. crombezi (Bourguignat, 1880) inhabits the Alpes-Maritimes (Falkner et al., 2002).

Remarks. In the most recent literature (Bank et al., 2001; Falkner et al., 2002) Corneola is regarded as a subgenus of Chilostoma, with four species. Two of these, viz. Corneola desmoulinsii (Fig. 1.24) and $C$. squamatinum, are included in this study. See Corneola in Schileyko (2013) for details regarding the shell and genital morphology. The accessory glands are undivided (Fig. 4.5) 
Genus Cylindrus Fitzinger, 1833 (monotypic) Type species: Pupa obtusa Draparnaud, 1805

Molecular data. The phylogeny reconstructions for the combined datasets show strong support for a sistergroup relation between Cylindrus and Arianta $(\mathrm{PP}=$ 1.0, Figs 1, 2, S5, S6). Only the phylogeny based on COI fails to indicate that these taxa are sister-groups, thus sharing a unique common ancestor. The sequence divergence between Cylindrus and Arianta is ca. 19\% for $C O I$ and up to $25 \%$ for $C y t B$ (table 4).

Age. The common ancestor of Arianta and Cylindrus is estimated to have diverged at ca. 47.6-46.4 MYA (Figs 2, S5).

Distribution. Endemic to the Austrian Alps (between 1600 and $2500 \mathrm{~m}$ ), known from Oberösterreich, Niederösterreich, Salzburg, Steiermark and Kärnten.

Remarks. Among the (sub)genera of Ariantinae that can be distinguished by shell-morphology, Cylindrus is the most distinctive because the shell is cylindrical and much higher than broad (Fig. 3.3). Its sister-group, the genus Arianta, is characterized by much larger shells that vary in shape between flattened and globular. This close relationship, which is surprising in view of the morphological data, was reported by Groenenberg et al. (2012) and later on confirmed by Cadahia et al. (2013). Despite the long geological history of Cylindrus that is indicated by the molecular data and is also suggested by its aberrant shell morphology, no clear fossil representatives of this genus, or forms that are transitional in shell-shape, are known from before the Würm (Zilch, 1960; Frank, 2006). See Cylindruini in Schileyko $(2006,2013)$ for details regarding the structure of the genital tract. Cylindrus has undivided accessory glands (Fig. 4.2).

Genus Delphinatia Hesse, 1931

Type species: Helix alpina Michaud, 1831

Molecular data. Delphinatia fontenillii alpina (Michaud, 1831), and D. glacialis (Férussac, 1832) together are monophyletic ( $\mathrm{PP} \geq 0.93$, Figs $2, \mathrm{~S} 1, \mathrm{~S} 2, \mathrm{~S} 6)$ and form a clade with Drobacia and Vidovicia in the phylogeny reconstructions for the combined datasets $(0.5 \geq \mathrm{PP} \geq 0.86$, Figs $1,2, \mathrm{~S} 5, \mathrm{~S} 6)$. However, in the trees based on individual markers, this clade is only observed with $H 3$ ( $\mathrm{PP}=0.8$, Fig. S1). There is no consensus regarding the sister-group relations of these three taxa. Only of D.f. alpina sufficient sequence data were obtained to include it in the phylogeny re- constructions of the stringent dataset (Figs 1, S5).

Age. The most recent common ancestor of the combined group Delphinatia-Drobacia-Vidovicia is estimated at ca. 59.1-53.3 MYA (Figs 2, S5); that of Delphinatia is estimated at ca. 17.5 MYA (Fig 2).

Distribution. French Alps (departments of HautesAlpes, Haute-Savoie, Isère and Savoie) to the adjacent Italian Alps (Alpi Cozie and Graie) (Gavetti et al., 2008).

Remarks. Delphinatia is considered a subgenus of Chilostoma by Bank et al. (2001), but has been classified as a subgenus of Campylaea as well (Zilch, 1960). Only two species are generally recognized in Delphinatia, viz. D. fontenillii (Michaud, 1829), and D. glacialis, which are both included in this study. Falkner $e t$ al. (2002) distinguished D. f. fontenillii and D. f. alpi$n a$ (Fig. 3.11) next to the monotypic D. glacialis. See Campylaea (Delphinatia) and Chilostoma (Delphinatia) in Schileyko $(2006,2013)$ for details regarding the shell and genital morphology. The accessory glands (Fig. 4.12) are undivided, or one of them is split for up to $25-50 \%$ of its length.

Subgenus Dinarica Kobelt, 1902, genus Dinarica Type species: Helix pouzolzii Deshayes, 1830

Molecular data. Two subgenera of Dinarica can be recognized, viz. Dinarica (Dinarica) and D. (Sabljaria). In this study, the former taxon is represented by D. (Dinarica) pouzolzii (Fig. 3.22) and D. (D.) serbica Kobelt, 1872. Dinarica (Dinarica), as well as the genus itself, are shown to be monophyletic ( $\mathrm{PP}=1$, Figs $1,2, \mathrm{~S} 5, \mathrm{~S} 6)$. The $C O I$ sequence divergence between $D$. (Dinarica) serbica and D. (Dinarica) pouzolzii is about 10\% (Table 4).

The phylogenies based on the concatenated datasets slightly differ regarding to the position of Dinarica. Figures 2, S5 and S6 suggest a sister-group relationship between Dinaricia and Liburnica $(0.44 \leq \mathrm{PP}$ $\leq 0.78)$. In these figures Dinarica - Liburnica has a sister-group relation with the clade Kollarix - Pseudotrizona - Cattania - Thiessea - Josephinella. Basically Fig 1 shows the same topology, but here Liburnica is the sister-group of the latter genera including Dinarica.

Age. The most recent common ancestor of Dinarica is estimated at ca. 37.1-36.2 MYA (Figs 2, S5).

Distribution. Along the NE coast of the Adriatic sea, in SE Croatia, Bosnia-Herzegowina, S Servia, Montenegro, Kosovo, Albania, the western border of 
Macedonia and NW Greece. Dinarnica (D.) pouzolzii and $D$. (D.) serbica have partially overlapping ranges. Where the former dominates the coastal region of Montenegro, the latter occurs more inland.

Remarks. See Campylaea (Dinarica) and Chilostoma (Dinarica) in Schileyko $(2006,2013)$ for details regarding the shell and genital morphology. Dinarica (Dinarica) has split accessory glands (Fig. 4.23).

Genus Drobacia Brusina, 1904

Type species: Helix banatica Rossmässler, 1838

Molecular data. Both Drobacia species, viz. D. banatica (Fig. 3.26) and D. cf maeotica Wenz, 1926 (in Krejci and Wenz, 1926), are included in this study. The taxon is shown to be monophyletic ( $\mathrm{PP}=1.0$ Figs 1,2 , S5, S6). The position of Drobacia within the subfamily Ariantinae is still unclear. The phylogeny reconstructions indicate that Drobacia forms a clade with Delphinatia and Vidovicia (see Delphinatia). Only the phylogeny for COI supports a sister-group relationship with Liburnica ( $\mathrm{PP}=0.86$; Fig. S2).

Age. For an age estimation of the most recent common ancestor of Drobacia, Delphinatia and Vidovicia, see Delphinatia. Drobacia banatica and D. cf maeotica are estimated to have diverged ca. 8.7-7.8 MYA (Figs 2, S5).

Distribution. W and SW Romania and locally in E Hungary. In the Pleistocene Drobacia reached as far as the Harz Mts in Thüringen, Germany (Jaeckel, 1962).

Remarks. See Helicigona (Drobacia) and Drobacia in Schileyko $(2006,2013)$ for details regarding the shell and genital morphology. Drobacia has undivided accessory glands (Fig. 4.9).

Genus Faustina Kobelt, 1904

Type species: Helix faustina Rossmässler, 1835

Molecular data. Faustina is shown monophyletic in all our phylogeny reconstructions ( $\mathrm{PP} \geq 0.99$ ), but $C y t B$ and $16 S$ sequences were only obtained for subspecies of $F$. faustina. Consequently the monophyly of the genus could only be assessed with the data for $\mathrm{H3}$ and COI. Sequence divergences within Faustina are generally large; between $F$. faustina orba (von Kimakowicz, 1890) and F. kiralikoeica (von Kimakowicz, $1890)$ the sequence divergence for $C O I$ is $17.4 \%$. Even between the alleged subspecies $F$. f. faustina (Ross- mässler, 1835) and F.f. associata (Rossmässler, 1835) divergences reach up to $10.7 \%$ (Table 4). A sister-group relationship between Faustina and Kosicia is shown with the phylogeny reconstructions for the concatenated datasets ( $\mathrm{PP} \geq 0.95$; Figs 1, 2, S5, S6). The phylogeny for $H 3$ indicates Faustina as the sister-group of all other Ariantinae, but this is not supported by any of the other phylogeny reconstructions.

Age. The most recent common ancestor of the investigated Faustina specimens is estimated at ca. 13.411.3 MYA. The split between Faustina and Kosicia is estimated at 56-51.7 MYA (Figs 2, S5).

Distribution. The Carpathian Mts, E Czech, Slovakia, S Poland, W Ukraine and Romania; also in NE Hungary. Faustina faustina (Fig. 3.12) has the widest distribution, $F$. rossmaessleri (Pfeifer, 1848) and $F$. cingulella (Rossmässler, 1837) are mainly found in Slovakia, F. barcensis (von Kimakowicz, 1890) and $F$. kiralikoeica are found in Romania.

Remarks. There are at least 5 Faustina species, 3 of which are included in this study, viz. the nominate subspecies of $F$. faustina, two additional subspecies $[F$. faustina associata and $F$. faustina orba], and $F$. kiralikoeica. See Campylaea (Faustina) and Faustina in Schileyko $(2006,2013)$ for details regarding the shell and genital morphology. In Faustina both types of accessory glands occur. Faustina cingulella and F. rossmaessleri have undivided glands, whereas they are split up to half their length in F. faustina (Fig. 4.19), F. barcensis and F. kiralikoeica.

Genus Helicigona Férussac, 1821

Type species: Helix lapicida Linnaeus, 1758

Molecular data. In the past this generic name has been used for many taxa of the Ariantinae (Hesse, 1931; Knipper, 1939; Zilch, 1960; Subai, 1984). None of our phylogeny reconstructions support these views. The phylogeny reconstructions of the concatenated datasets indicate that Causa and Isognomostoma together, are most likely the sister-group of Helicigona (group A; Figs 1,2, S5, S6). The monophyly of the two alleged subspecies of $H$. lapicida is beyond dispute ( $\mathrm{PP}=1.0$; Figs 1, 2, S1-S6). $C O I$ and $C y t B$ sequence divergences within each subspecies are less than $2.5 \%(n=4)$, but between both subspecies they reach up to $12 \%$ and $14 \%$, respectively.

Age. The only fossil that can be indisputably assigned to any of the currently recognized Ariantinae is a representative of Helicigona (see Nordsieck, 2014 


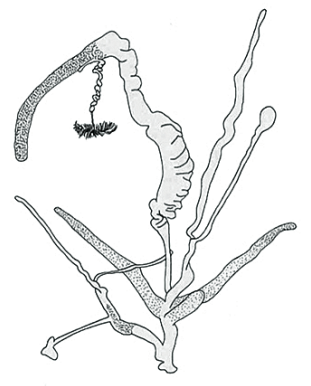

1. Arianta arbustorum

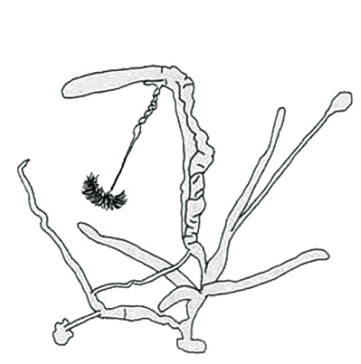

5. Corneola squamatinum

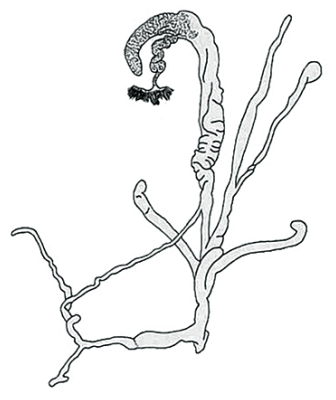

2. Cylindrus obtusus

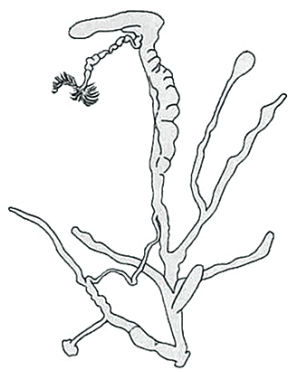

6. Helicigona lapicida

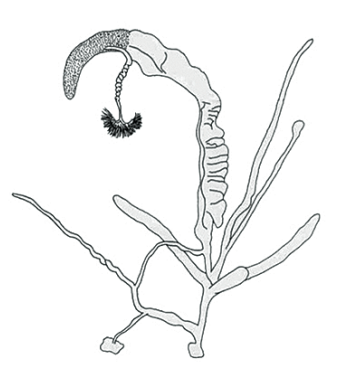

3. C. (Chilostoma) adelozona

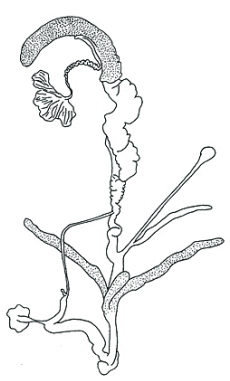

7. Causa

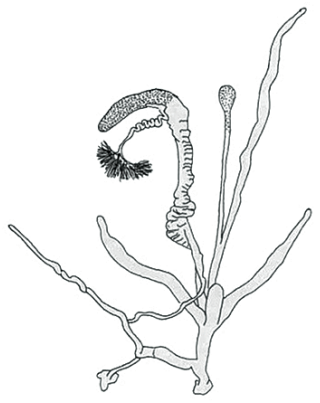

4. C. (Cingulifera) cingulatum

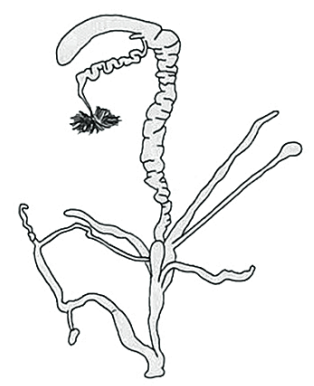

8. Isognomostoma

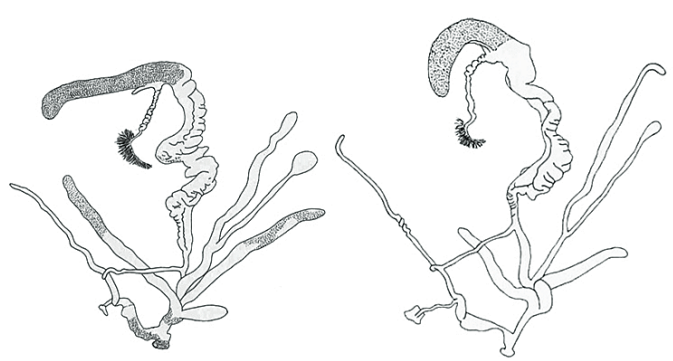

\section{Drobacia} banatica

10. Campylaeopsis moellendorffii holosericea

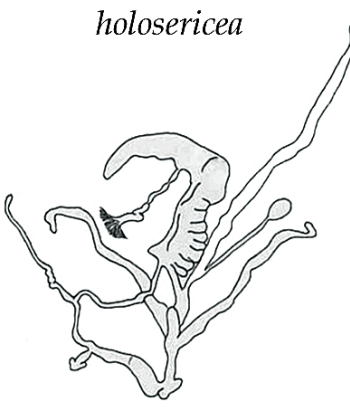

11. Vidovicia caerulans isognomostomos

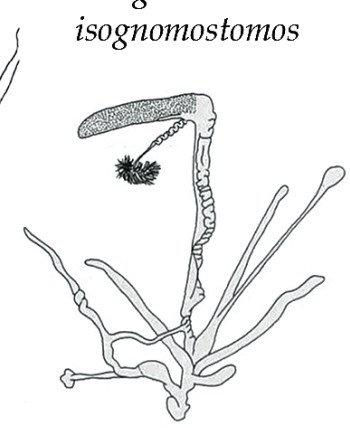

12. Delphinatia fontenillii alpina

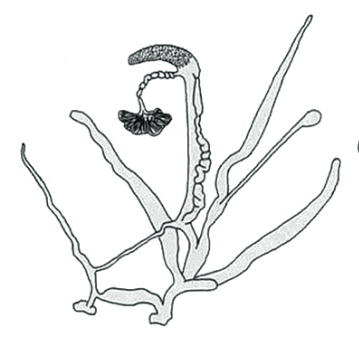

13. Kosicia intermedia

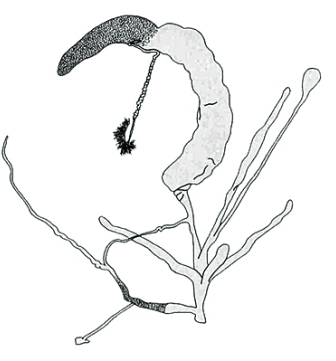

14. C. (Campylaea) planospira

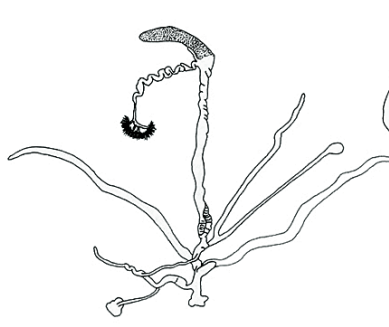

15. Kollarix kollari

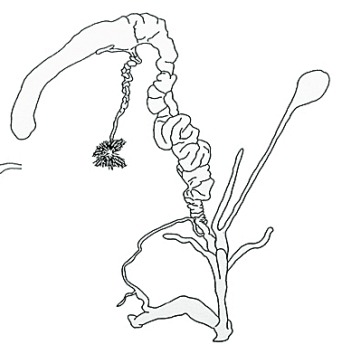

16. Thiessea sphaeriostoma

Fig. 4. Genital anatomy for most of the currently recognized genera of Ariantinae.The simplified diagram of the genital morphology was reproduced and adapted from Koene and Schulenburg (2005; Creative Commons Attribution License 2.0). 


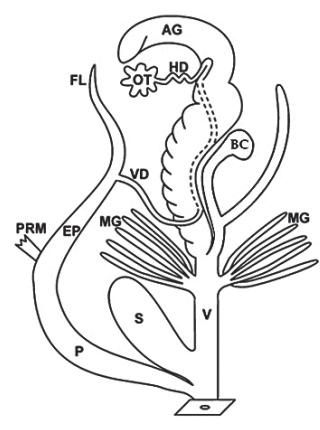

Genital morphology

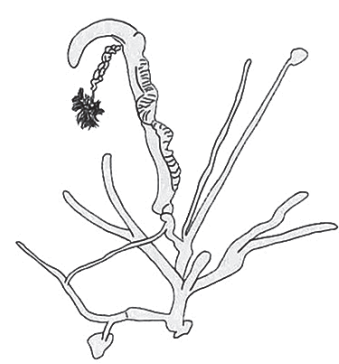

19. Faustina faustina
$A G=$ albumen gland

$B C=$ bursa copulatrix

$B T D=$ diverticulum

$E P=$ epiphallus

$F L=$ flagellum

$H D=$ hermaphroditic duct

$M G=$ accessory glands

$O T=$ ovotestis

$P=$ penis

$P R M=$ penis retractor muscle

$S=$ dart sac (bursa telae)

$V=$ vagina

$V D=$ vas deferens

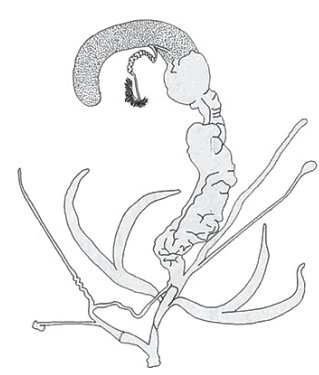

20. L. (Liburnica) setosa

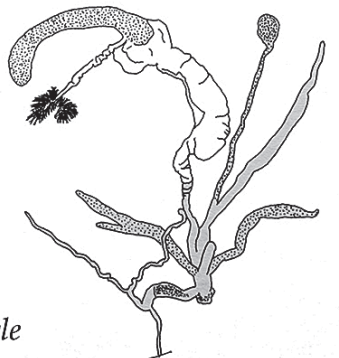

17. L. (Superba) skipetarica

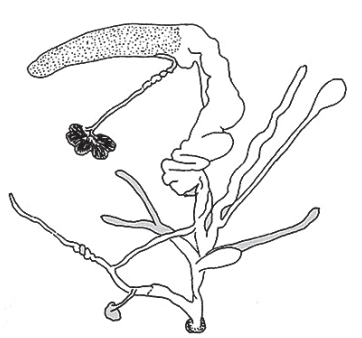

18. Josephinella reischuetzi

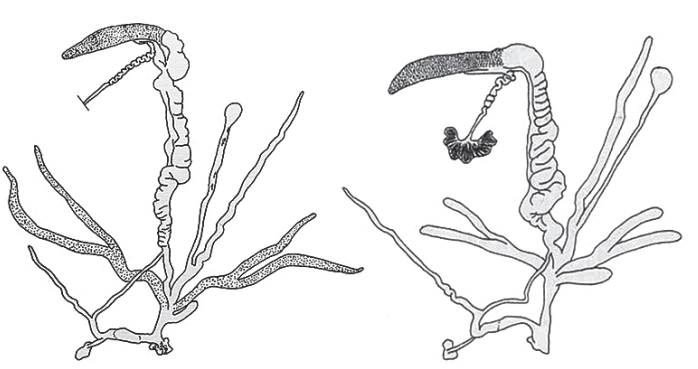

21. L. (Superba) kulmakana
22. Josephinella hemonica

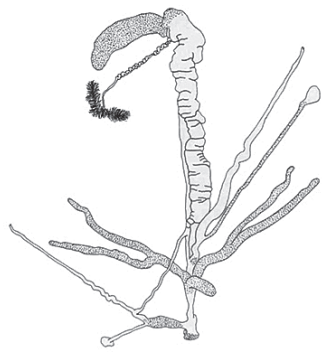

23. D. (Dinarica) pouzolzii

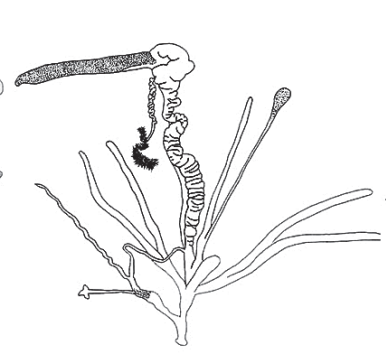

24. D. (Sabljaria) stenomphala

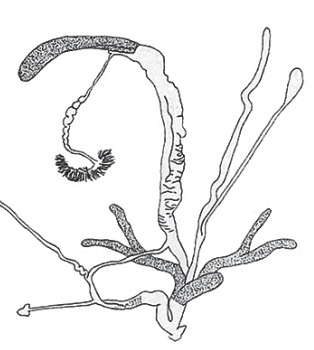

25. C. (Oricampylaea) ljubetenensis

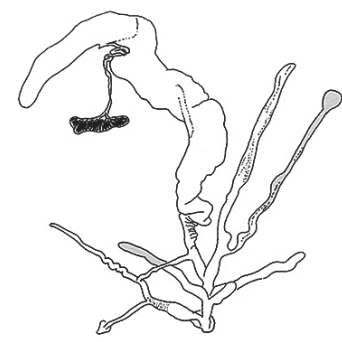

26. Josephinella vikosensis

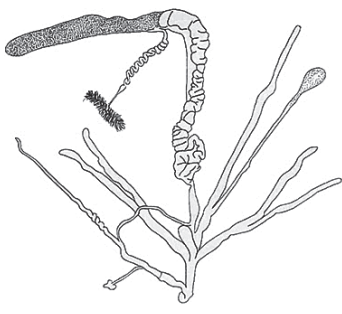

27. C. (Cattania) trizona

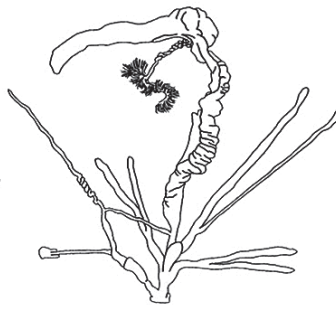

28. Pseudotrizona inflata

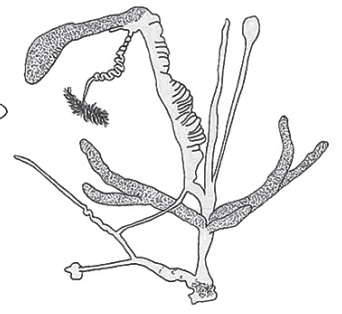

29. C. (Ariantopsis) pelia

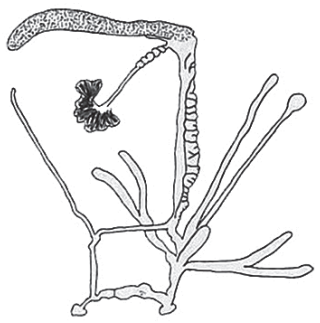

30. C. (Wladislawia) polinskii 
and references therein) from the Late Burdigalian ( 17.5-16.0 MYA). This date was the only calibration point used in our BEAST analyses. The split between Helicigona and the lineage Causa-Isognomostoma is estimated at ca. 62.6-61.2 MYA (Figs 2, S5).

Distribution. The nominate subspecies is widely distributed in $\mathrm{W}$ and $\mathrm{N}$ Europe, from S Scandinavia and central England to the south up to $\mathrm{S}$ France, to the east up to Czech and W Poland. Helicigona lapicida andorrica (Bourguignat, 1876) is restricted to the eastern Pyrenees.

Remarks. Helicigona is considered a monotypic genus with only two clearly differentiated subspecies, viz. Helicigona l. lapicida (Fig. 3.25) and H. l. andorrica, which are both included in this study. See Helicigona (Helicigona) and Chilostoma (Helicigona) in Schileyko $(2006,2013)$ for details regarding the shell and genital morphology. The accessory glands are undivided (Fig. 4.6).

Genus Isognomostoma Fitzinger, 1833 (monotypic) Type species: Helix personata Lamarck, 1792 [= Isognomostoma isognomostomos (Schröter, 1784)]

Molecular data. Isognomostoma isognomostomos (Fig. 3.4) and Causa holosericea (Fig. 3.5) have long been regarded as congeneric. All phylogeny reconstructions, except the one based on $H 3$ (Fig. S4), explicitly show Causa and Isognomostoma together as a monophyletic group ( $\mathrm{PP} \geq 0.99$; Figs 1, 2, S2-S6). See also the paragraph on Causa.

Distribution. Mountains of central Europe, $\mathrm{S}$ of the line Eifel, Sauerland and the Harz Mts. From E France eastwards in Zwitserland, Austria, N Italy, Slovenia, Croatia, Czech, Slovenia, S Poland (Carpathians), NE Hungary and Rumania.

Remarks. See Isognomostoma in Schileyko (2006, 2013) for details regarding the shell and genital morphology. The accessory glands are undivided (Fig. 4.8).

Genus Josephinella Haas, 1936

Type species: Helix hemonica Thiesse, 1884 (Fig. 3.34)

Molecular data. Based on 11 included species (2 undescribed; Table S7), Josephinella is considered a monophyletic group (PP = 1.0; Figs 1, 2, S2-S6). The phylogeny reconstructions for the combined datasets show Thiessea as the sister-group of Josephinella (PP
$=1.0 ;$ Figs 1, 2, S5, S6). Josephinella reischuetzi (Subai, 1990) and J. vikosensis (Subai, 1990) together, which were once classified in Superba by Subai and Fehér (2006) are shown to belong to Josephinella (PP $\geq 0.87$; Figs S1, S2).

Age. The most recent common ancestor of Josephinella (based on four taxa) is estimated at ca. 22-21.6 MYA (Figs S5, 2).

Distribution. Southern half of Albania, the SW border area of Macedonia (FYROM), the Ionian islands, mainland Greece and the Peloponnese.

Remarks. With at least 18 named species, and more than 10 still to be described (Subai, in prep.), Josephinella is the most speciose genus of the Ariantinae. For this study 11 species were included.

See Helicigona (Josephinella) and Chilostoma (Josephinella) in Schileyko $(2006,2013)$ for details regarding the shell and genital morphology. The accessory glands are generally split from halfway up to $2 / 3$ of their length (Fig. 4.22); specimens with one or both glands undivided (J. vikosensis and J. reischuetzi; Fig. 4.26 and Fig. 4.18) or trifurcate accessory glands are rare ( $\mathrm{n}=60: 4$ undivided, 56 split, of which 3 specimens had only one divided gland).

\section{Genus nov. Kollarix}

Type species: Helix kollari Pfeiffer, 1856 (monotypic)

Diagnosis. The diagnosis of this monotypic subgenus is by definition identical with that of its type species, i.e. Kollarix kollari (Pfeiffer, 1856). Kollarix gen. nov. is also differentiated by a unique combination of nucleotides in the 655 bp COI sequence obtained with general barcoding primers (Folmer, 1994) at the following relative positions: $16 \mathrm{C}, 67 \mathrm{~A}, 68 \mathrm{~A}, 84 \mathrm{G}, 94 \mathrm{~T}$, 357 G, 475 A, 493 G, 556 G, 625 C.

Description. Shell strongly depressed, nearly discoid, with 43/4-51/4 whorls; umbilicus wide, measuring $1 / 5-1 / 6$ of the total shell width; corneous brown, with a brown spiral band in a whitish zone (see Welter-Schultes, 2012: 595, Helicigona kollari); surface finely granulated, with growth lines and hairs. Height 8.5-12.0 $\mathrm{mm}$; width 18.5-27.0 $\mathrm{mm}$. The accessory glands are always undivided (Fig. 4.15). Stimulator broad, flattened, filling the genital atrium and reaching far into the vagina; an extension ends at the insertion of the penis. Penial papilla small, conical, with fine transverse ridges and an obtuse apex with a short, transverse, slit-like pore. Secondary ureter closed for 0.5 $1.0 \mathrm{~mm}$ and open for the remaining $2-3 \mathrm{~cm}$. 
Molecular data. Genetically, Kollarix kollari cannot be assigned to Liburnica (Subai, 2002), nor any other of the described genera. Kollarix is shown to be a separate lineage within the Ariantinae, which is more closely related to Pseudotrizona gen. nov., than to Liburnica Kobelt, 1904 (Figs 1, 2, S5, S6).

Age. The most recent common ancestor of Kollarix and the clade (Pseudotrizona - Cattania - Josephinella - Thiessea) is estimated at ca. 55.9-55.8 MYA (Figs 2, S5).

Distribution. Endemic to Serbia, $\mathrm{S}$ of the Donau up to Aleksinac, between Šabac and Bor districts.

Remarks. Helix kollari Pfeiffer, 1856 (Fig. 3.19) has been classified in Campylaea (by Tomić, 1959), in Helicigona (by Knipper, 1939; Maassen, 1985) and most recently in Liburnica (by Subai, 2002; Bank, 2012). In his treatise on Liburnica, Subai (2002) hypothesized that Kollarix kollari might be the oldest representative of Liburnica. Since Kollarix kollari cannot be assigned to any of the described genera, neither genetically nor morphologically, it is here given generic status. The name Kollarix has been used by Groenenberg et al. (2012) and Schileyko (2013: 146), but in both cases with the explicit note that this was not for purposes of zoological nomenclature.

Derivatio nominis. Kollarix refers to kollari.

Genus Kosicia Brusina, 1904

Type species: Helix intermedia Pfeiffer, 1828

Molecular data. Kosicia is usually regarded as a subgenus of Chilostoma (Zilch, 1960; Bank et al., 2001), but should be given generic status based on our phylogeny reconstructions. Its three species, viz. Kosicia ambrosi (Strobel, 1852) (Fig. 3.15), K. intermedia (Fig. 3.16) and K. ziegleri (Rossmässler, 1836) (Fig. 3.17) form a monophyletic group (PP $\geq 0.94$; Figs 1,2 , S1S6). Kosicia ambrosi, which is much smaller than the other two species, is the sister-group of $K$. intermedia and $K$. ziegleri together (Figs 1, 2, S2, S3, S5, S6). The phylogeny reconstructions for the concatenated datasets show Faustina as the sister-group of Kosicia (PP $=1.0 ;$ Figs 1, 2, S5 and S6).

Age. The most recent common ancestor of Kosicia is estimated at ca. 30.1-28.0 MYA (Figs 2, S5); that of $K$. intermedia and $K$. ziegleri is estimated at ca. 7 MYA (Figs 2, S5).

Distribution. Kosicia intermedia is most widely distributed; it occurs in NE Italy, S Austria (Kärnten), NE Italy, Slovenia and NW Croatia. Kosicia ambrosi has the smallest range; it is endemic to E Trentino and the Prealps of Veneto (Italy). Kosicia ziegleri occurs in $\mathrm{S}$ Kärnten (Austria) and in the border area between Italy and Slovenia.

Remarks. All three known Kosicia species were included in this study. See Helicigona (Kosicia) and Kosicia in Schileyko $(2006,2013)$ for details regarding the shell and genital morphology. The accessory glands are always undivided (Fig. 4.13).

Subgenus Liburnica Kobelt, 1904, genus Liburnica Type species: Helix setosa Férussac, 1832 (Fig. 3.20)

Molecular data. Liburnica has been regarded a subgenus of Campylaea (Zilch, 1960) and Chilostoma by Bank et al. (2001), but none of our phylogeny reconstructions indicate a close relationship between any of these taxa. Liburnica respresents a distinct, monophyletic lineage ( $\mathrm{PP}=1.0$; Figs 1, 2 and S1-S6), which includes Superba (Subai and Fehér, 2006) according to $\mathrm{H} 3$ and $\mathrm{COI}$ sequences (Figs S1, S2; see Superba). The position of Liburnica is only partly resolved; our data hint at a sister-group relation with Dinarica $(0.44 \leq \mathrm{PP}$ $\leq 0.78$; Figs 2, S5, S6; see Dinarica). Only the phylogeny based on $C O I$ explicitly supports another sistergroup relation; see Drobacia. Six species of Liburnica (Liburnica) could be investigated. The subgenus is shown as a monophyletic group in the phylogeny based on $C O I$ ( $\mathrm{PP}=0.75$; Fig. S2). $C O I$ sequence divergences within $L$. (Liburnica) range from $6.3 \%$ to $3.4 \%$. Between L. (Liburnica) and L. (Superba) the COI sequence divergence is $\leq 6.9 \%$ (Table 4 ).

Age. The most recent common ancestor of Liburnica and Dinarica is estimated at ca. 53.2-51.4 MYA; that of Liburnica (Liburnica) is estimated at ca. 5.93.1 MYA (Figs 2, S5).

Distribution. Mts. along the NE coast of the Adriatic Sea in Croatia, Bosnia-Herzegowina, Montenegro, Kosovo, Albania, W Macedonia, southwards to Epirus in NW Greece.

Remarks. With over 15 described species, Liburnica is among the most speciose genera of Ariantinae. Conchologically Liburnica is quite variable (Fig. 3.20, 3.23). The 6 species used in this study (10 including Super$b a)$, suggest that these forms radiated rapidly $(<\sim 6$ MYA; see Age). See Subai (2002) and Schileyko (2013) for details regarding the shell and genital morphology. The upper $1 / 3$ to $2 / 3$ of the accessory glands in L. (Liburnica) are generally split (Fig. 4.20); occasionally one (Fig. 4.17) or both glands are undivided. 
Subgenus nov. Oricampylaea, genus Campylaea Type species: Faustina (Campylaea) illyrica Stabile, 1864

Diagnosis. Oricampylaea subgen. nov. is differentiated by a unique combination of nucleotides in the 655 bp COI sequence obtained with general barcoding primers (Folmer, 1994) at the following relative positions: $88 \mathrm{~T}, 187 \mathrm{~T}, 220 \mathrm{~T}, 301 \mathrm{~T}, 385 \mathrm{~A}, 409 \mathrm{C}, 556 \mathrm{~T}$, $575 \mathrm{~T}, 578 \mathrm{C}, 650 \mathrm{C}$.

Molecular data. This clade ( $\mathrm{PP}=1.0$; Fig. S2) consists of at least the species Campylaea (Oricampylaea) illyrica and C. (Oricampylaea) lefeburiana (Férussac, 1821). After our $\mathrm{H3}$ sequence, Helicigona (Arianta) ljubetenensis Wagner, 1914 (in Sturany and Wagner, 1914), which was regarded as a subspecies of Cattania (C.) trizona by Knipper (1939) and Bank (2012), has to be added as a third species.

Age. Based on the intraspecific divergence in $C$. (Oricampylaea) illyrica, the most recent common ancestor of $C$. (Oricampylaea) is estimated at, at least, ca. 19-17.9 MYA (Figs 2, S5).

Distribution. Southern Germany (introduced), southern Austria, from Slovenia southwards to W and N Croatia. Campylaea (Oricampylaea) illyrica also occurs along the SW Hungarian border, in N Serbia and in SW Romania. Campylaea (Oricampylaea) ljubetenensis is restricted to the Šar Mts (between Kosovo and NW Macedonia).

Remarks. The phylogeny reconstructions based on $H 3$ and $C O I$ show a clade within Campylaea that separates $C$. (Oricampylaea) illyrica and $C$. (Oricampylaea) lefeburiana from C. (Campylaea) planospira. Here we denoted this group Oricampylaea subgen. nov., because this clade persists even in case the untimely inclusion of $C$. (Oricampylaea) ljubetenensis would turn out to be incorrect. Observing C. (Oricampylaea) ljubetenensis in a clade (data for $\mathrm{H} 3$ only) with $C$. (Oricampylaea) illyrica is surprising both morphologically as well as geographically; in shell shape $C$. (Oricampylaea) ljubetenensis resembles $C$. (Cattania) trizona more than C. (Oricampylaea) illyrica, whereas it occurs ca. $250 \mathrm{~km}$ south of the distribution area of the latter species. Future research has to show if the provisional assignment of $C$. ljubetenensis to $C$. (Oricampylaea) will uphold and whether Campylaea hirta (Menke,1830), C. macrostoma (Rossmässler, 1836), C. schlaerotricha (Bourguignat, 1870), and $C$. sadleriana (Rossmässler, 1838) should be assigned to this new subgenus as well. The accessory glands for $C$. (Oricampylaea) lefeburiana and C. (Oricampylaea) ljubetenensis (Fig. 4.25) are split, whereas those for $C$. (Oricampylaea) illyrica are undivided (Knipper, 1939). The name Ljubotenia has been used for C. (Oricampylaea) ljubetenensis by Groenenberg et al. (2012) and Schileyko (2013: 146), but in both cases with the explicit note that this was not for purposes of zoological nomenclature.

Derivatio nominis. The epithet Oricampylaea is used for a group of oriental Campylaea species, which cannot yet be diagnosed with morphological characters.

\section{Genus nov. Pseudotrizona}

Type species: Helix inflata Kobelt, 1876 (monotypic)

Diagnosis. The diagnosis of this monotypic subgenus is by definition identical with that of its type species, i.e. Pseudotrizona inflata (Kobelt, 1876). Shell light corneous with three brown spiral bands and a narrow umbilicus. Pseudotrizona gen. nov. is also differentiated by a unique combination of nucleotides in the 655 bp COI sequence obtained with general barcoding primers (Folmer, 1994) at the following relative positions: $22 \mathrm{~A}, 181 \mathrm{~A}, 265 \mathrm{G}, 271 \mathrm{G}, 304 \mathrm{~A}, 325 \mathrm{~A}, 413 \mathrm{C}$, $481 \mathrm{G}, 616$ A, 649 C.

Description. Shell depressed conical, whitish to light corneous, with three brown spiral bands (WelterSchultes, 2012: 594, Helicigona inflata). Surface with growthlines only. With 43/4-51/2 whorls; umbilicus narrow, measuring c. $1 / 10$ of the total shell width. Height 10.5-18.0 mm; width 20.3-31.5 mm.

The accessory glands (Fig. 4.28) may be split for $1 / 3$ to $1 / 2$ of their length, but occasionally specimens with both an undivided and a split glandula occur as well. Stimulator more or less rounded triangular, prominently protruding obliquely in the central part of the genital atrium. Penial papilla slender conical, sometimes narrowed in the middle, with fine transverse ridges. Secondary ureter closed for 0.1-0.15 $\mathrm{mm}$ and open for the remaining $3.5-4.0 \mathrm{~cm}$.

Molecular data. In the phylogeny reconstructions based on the concatenated datasets this species is always the sister-group of the clade Cattania-Josephinella-Thiessea (PP = 1.0; Figs 1, 2, S5, S6). None of the phylogenies show a species group exclusively consisting of Pseudotrizona and Cattania, thus Pseudotrizona inflata is not a species of Cattania.

Age. The lineage that gave rise to Pseudotrizona is estimated to have diverged from the common ancestor of Cattania-Josephinella-Thiessea at ca. 53.4-48.8 MYA (Figs 2, S5). 
Distribution. N Albania, Montenegro, Kosovo, SW Serbia.

Remarks. Pseudotrizona inflata (Kobelt, 1876) has long been considered a subspecies of Cattania trizona, which was classified in Campylaea by Sturany and Wagner (1914), and in Helicigona by Knipper (1939) and Subai (1995).

Derivatio nominis. The epithet Pseudotrizona refers to the former incorrect classification of the type species as a subspecies of Cattania (C.) trizona.

Subgenus Sabljaria Brusina, 1904 (monotypic), genus Dinarica

Type species: Helix stenomphala Menke, 1830

Molecular data. All phylogenies based on the concatenated datasets depict Dinarica (Sabljaria) and D. (Dinarica) as a monophyletic group, see Dinarica (genus-level sister-group relations are also discussed). The subgenera D. (Sabljaria) and D. (Dinarica) are genetically clearly different. The $C O I$ and $C y t B$ sequence divergences between $D$. (Sabljaria) and D. (Dinarica) are $16.3 \%$ and $23.8 \%$, respectively (Table 4).

Age. See D. (Dinarica) for the estimated age of the genus.

Distribution. Endemic to the Velebit Mts along the coast of Croatia.

Remarks. Dinarica (Sabljaria) differs from D. (Dinarica) both conchologically (Fig. 3.21, 3.22) and in genital anatomy (Fig. 4.23, 4.24). These subgenera are allopatrically distributed. See Chilostoma (Sabljaria) in Schileyko (2013) for details regarding the shell and genital morphology. Dinarica (Sabljaria) has split accessory glands (Fig. 4.24).

Subgenus Superba Subai and Fehér, 2006, genus Liburnica

Type species: Helicigona skipetaricus [sic] Subai, 1995

Molecular data. No CytB or $16 S$ sequences were obtained for L. (Superba) and H3 does not discriminate between the alleged subgenera of Liburnica (Fig. S1). Therefore the taxonomic status of L. (Superba) could only be assessed with COI. The phylogeny based on that marker shows L. (Liburnica) as a monophyletic group $(n=4)$ and L. (Superba) as paraphyletic $(n=4)$. Which of these subgenera is monophyletic depends on the selected outgroup. A phylogeny in which both are monophyletic, was not obtained. Partly based on these results, Subai (2012) synonymized Superba with $\mathrm{Li}$ burnica. The COI sequence divergences within $\mathrm{L}$. (Superba) are less than $2.1 \%$ (Table 4).

Age. Due to missing data, L. (Superba) was not included in the BEAST analyses. Given the limited amount of sequence divergence within Liburnica (and the larger intraspecific divergence in L. (Liburnica); Table 4), we expect $L$. (Superba) not to be older than L. (Liburnica); see L. (Liburnica).

Distribution. Albania, Tomor and Kulmakës Mts.

Remarks. Liburnica (Superba) contains three species, viz. L. (S.) skipetarica (Subai, 1995) (Fig. 3.23), L. (S.) grisea (Subai and Fehér, 2006) and L. (S.) kulmankana (Subai and Fehér, 2006), which are all included in this study (for remarks on J. reischuetzi and J. vikosensis; see sub Josephinella). See Subai and Fehér (2006) for details regarding the shell and genital morphology. The accessory glands are generally split (Fig. 4.21), one gland undivided is also observed (Fig. 4.17).

Genus Thiessea Kobelt, 1904

Type species: Helix cyclolabris Deshayes, 1839 (in Férussac and Deshayes, 1819-1851)

Molecular data. Thiessea is generally considered a subgenus of Chilostoma (Zilch, 1960; Bank et al., 2001). This view cannot be accepted, since both taxa are not shown to be closely related in any of our phylogeny reconstructions. The data obtained for Thiessea are limited; for three out of the four included species, only $H 3$ sequences were obtained. The $H 3$ phylogeny indicates the four Thiessea species as a monophyletic group (PP $=0.49$; Fig. S1). A sister-group relation is shown between Thiessea and Josephinella (PP = 1.0; Figs 1, 2, S1, S5, S6).

Age. The most recent common ancestor of Thiessea and Josephinella is estimated at ca. 39-36.6 MYA (Figs 2, S5).

Distribution. Mainland SE Greece, NE Peloponnese, Aegean Islands and SW Turkey.

Remarks. With at least 16 species (of which only 4 included in this study), Thiessea is one of the larger genera of Ariantinae. See Helicigona (Thiessea) in Subai (1996) and Schileyko (2006), and Chilostoma (Thiessea) in Schileyko (2013) for details regarding the shell and genital morphology. The accessory glands are undivided (Fig. 4.16; Subai, 1996). 
Genus Vidovicia Brusina, 1904 (monotypic) Type species: Helix lacticina Rossmässler, 1837 [= Vidovicia caerulans (Pfeiffer, 1828)]

Molecular data. Vidovicia is shown in a clade with Delphinatia and Drobacia in all phylogeny reconstructions based on the concatenated datasets (Figs 1, 2, S5, S6); see Delphinatia. The sister-group relationships of the genera within this clade remain elusive. The phylogeny reconstructions based on $C O I$ and $C y t B$ are uninformative regarding the position of Vidovicia (Figs S2, S3). That for $\mathrm{H} 3$ shows the mentioned clade ( $\mathrm{PP}=0.8$; Fig S1), while $16 \mathrm{~S}$ supports a sistergroup relation between Vidovicia and Corneola $(\mathrm{PP}=$ 0.94; Fig. S4).

Distribution. The Dalmatian mountains along the Croatian coast, from the Velebit Mts to the Peljesac peninsula. Found only on limestone rocks, generally below $1400 \mathrm{~m}$.

Age. Based on the two included specimens of this monotypic genus, the origin of Vidovicia is estimated at ca. 8.6 MYA (Figs 2, S5). For an age estimation of the clade Delphinatia-Drobacia-Vidovicia, see Delphinatia.

Remarks. Vidovicia (Fig. 3.29) has a typical depressed shell with a pointed apex and a fine to nearly rib-like radial sculpture. See Vidovicia in Schileyko (2006, 2013) for details regarding the shell and genital morphology. The accessory glands are undivided (Fig. 4.11).
Subgenus Wladislawia Wagner, 1928, genus Cattania Type species: Campylaea polinskii Wagner, 1928

Molecular data. None of our results indicate a close relationship between Cattania (Wladislawia) and Campylaea, Faustina or Chilostoma (see remarks). Instead, our phylogeny reconstructions always show Wladislawia within the clade of Cattania $(0.75 \leq \mathrm{PP} \leq$ 1.0; Figs 2, S1, S2, S5, S6). Cattania (Ariantopsis) and C. (Wladislawia) are sister-groups; see Ariantopsis.

Age. The most recent common ancestor of $C$. (Wladislawia) and C. (Ariantopsis) is estimated at ca. 7.3 MYA (Fig. 2); see Ariantopsis.

Distribution. Endemic to the Pirin Mts of SW Bulgaria, where it is found only at high altitudes.

Remarks. Only two Cattania (Wladislawia) species are known, viz. C. (W.) polinskii (Fig. 3.31) and C. (W.) sztolcmani Wagner, 1928 (Fig. 3.32). The latter species, thus not the type species, is included in this study. Wladislawia has been considered a subgenus of Campylaea by Zilch (1960), of Faustina by Damjanov and Likharev (1975) and more recently of Chilostoma by Bank et al. (2001). See Campylaea (Wladislawia) and Chilostoma (Wladislawia) in Schileyko $(2006,2013)$ for details regarding the shell and genital morphology. The accessory glands are split in C. (W.) polinskii (Fig. 4.30) and undivided in C. (W.) sztolcmani. 
Table 5. Proposed classification of the subfamily Ariantinae. ${ }^{1}$ our data indicate that Campylaea ljubetenensis is not a subspecies of Cattania trizona.$^{2}$ genus provisionally retained based on $\mathrm{H3}$ data. ${ }^{3}$ subgenus represented by Chilostoma (Achatica) achates. ${ }^{4}$ genus (Brusina, 1904) reintroduced, but given subgeneric ranking. ${ }^{5}$ Kollarix kollari does not belong to Liburnica s.str. ${ }^{6}$ genus (Subai and Fehér, 2006) given subgeneric ranking. ${ }^{7}$ Pseudotrizona inflata does not belong to Cattania s.lat.

Family Helicidae subfamily Ariantinae

\begin{tabular}{|c|c|c|c|}
\hline Genera & Subgenera & Included [Estimated] number of species & \\
\hline Arianta & & 4 [5] one with 5 subspecies & \\
\hline \multirow[t]{2}{*}{ Campylaea } & Campylaea & 1 [1] single polytypic species? & \\
\hline & Oricampylaea subgen. nov. & $3[7]$ & 1 \\
\hline \multirow[t]{4}{*}{ Cattania } & Ariantopsis & $1[1]$ monotypic & \\
\hline & Cattania & $5[9]$ & \\
\hline & Cattaniella subgen. nov. & $2[?]$ & \\
\hline & Wladislawia & 1[ 2] type species not included & \\
\hline Campylaeopsis & & $1[1]$ monotypic & 2 \\
\hline Causa & & $1[1]$ monotypic & \\
\hline \multirow[t]{3}{*}{ Chilostoma } & Achatica subgen. nov & $1[1]$ single polytypic species? & 3 \\
\hline & Chilostoma & $3[4 ?]$ & \\
\hline & Cingulifera & $1[1]$ polytypic species & \\
\hline Corneola & & $2[4]$ & \\
\hline Cylindrus & & $1[1]$ monotypic & \\
\hline Delphinatia & & $2[2]$ & \\
\hline \multirow{2}{*}{ Dinarica } & Dinarica & $2[2]$ & \\
\hline & Sabljaria & $1[1]$ & 4 \\
\hline Drobacia & & $2[2]$ & \\
\hline Faustina & & $3[5]$ & \\
\hline Helicigona & & 1 [1] monotypic (two subspecies both included) & \\
\hline Isognomostoma & & $1[1]$ monotypic & \\
\hline Josephinella & & $11[20]$ & \\
\hline Kollarix gen. nov. & & $1[1]$ monotypic & 5 \\
\hline Kosicia & & $3[3]$ & \\
\hline \multirow{2}{*}{ Liburnica } & Liburnica & $6[15]$ & \\
\hline & Superba & $3[3]$ & 6 \\
\hline Pseudotrizona gen. nov. & & $1[1]$ monotypic & 7 \\
\hline Thiessea & & $4[16]$ & \\
\hline Vidovicia & & $1[1]$ & \\
\hline
\end{tabular}




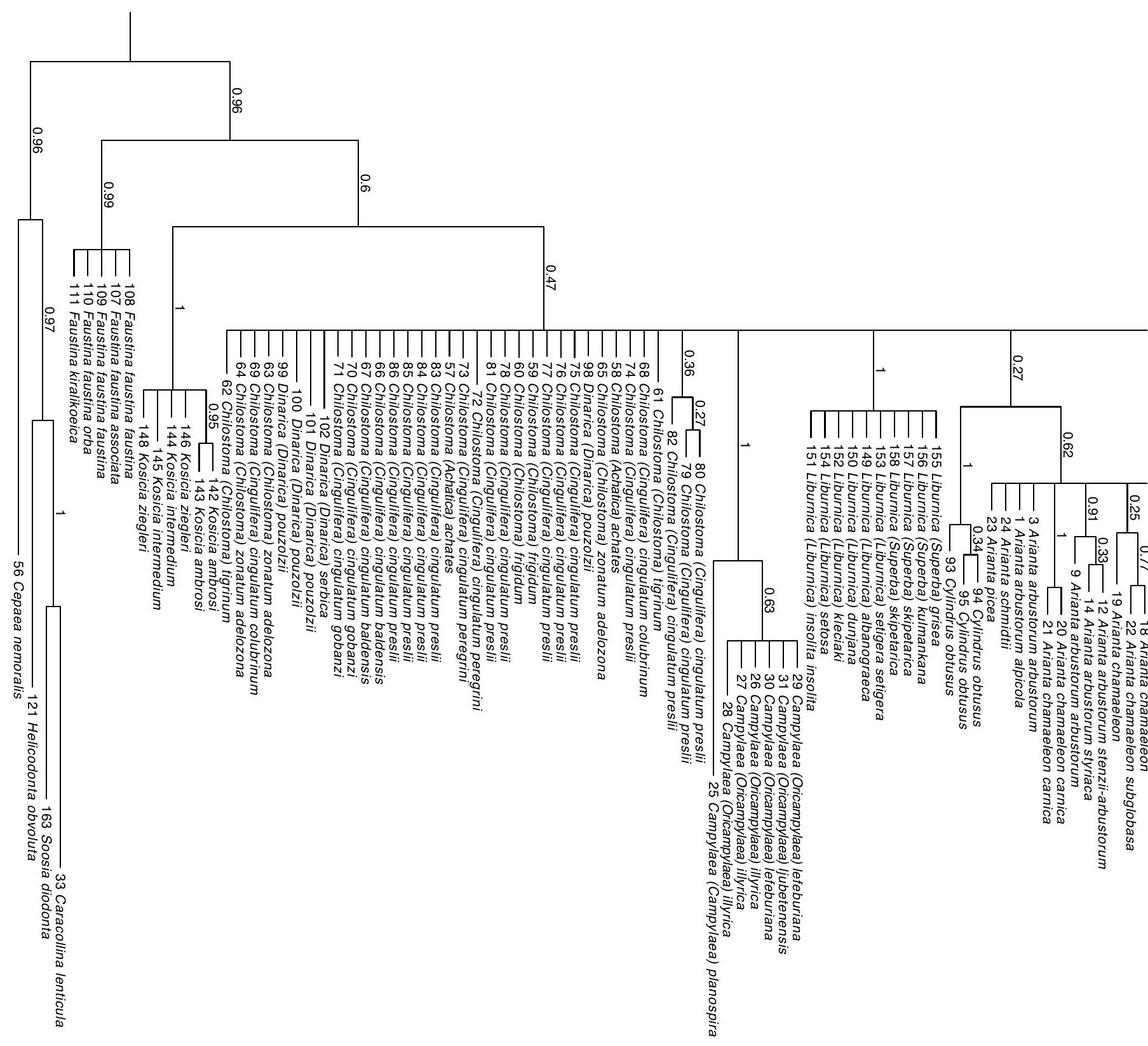




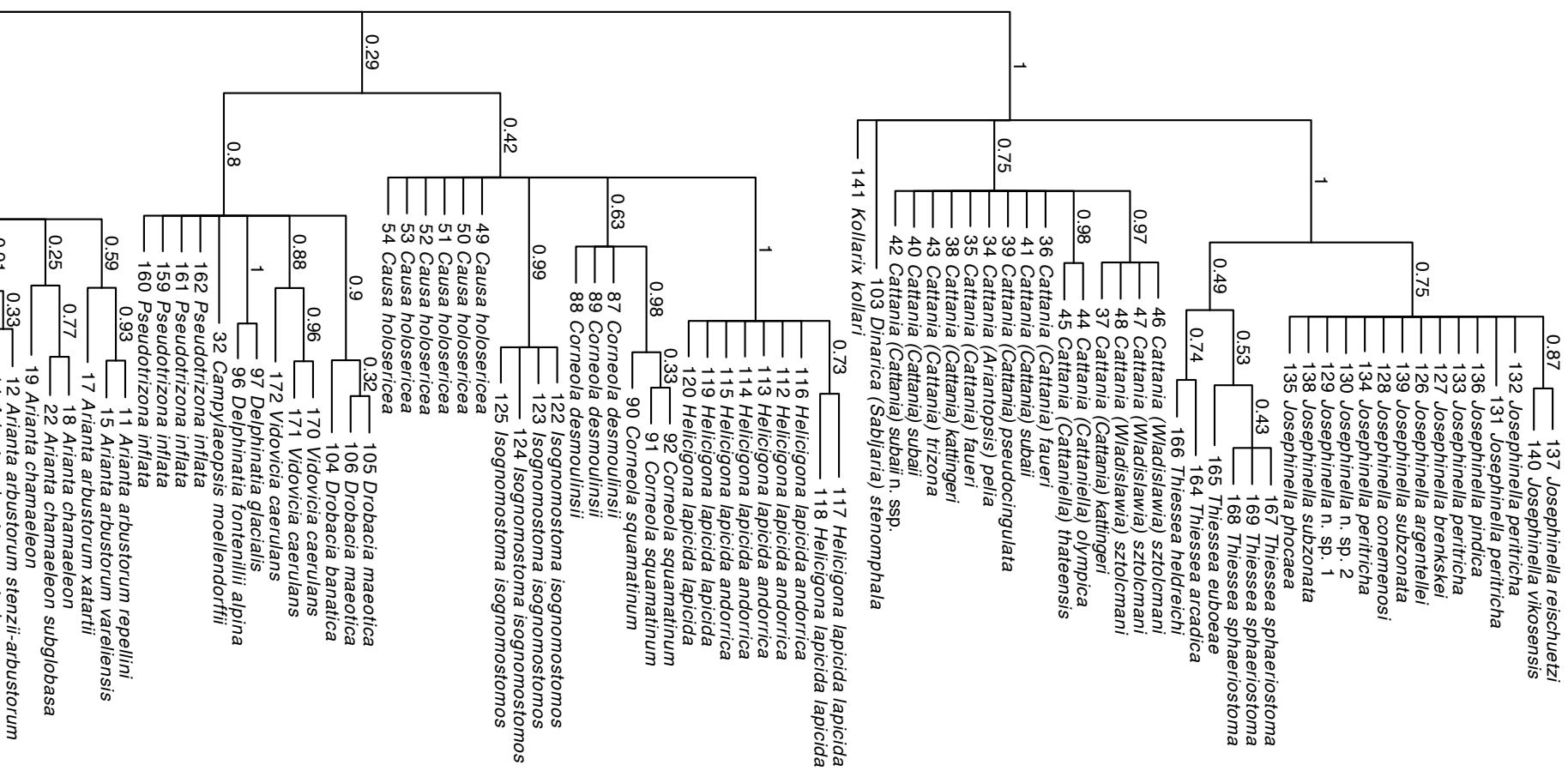




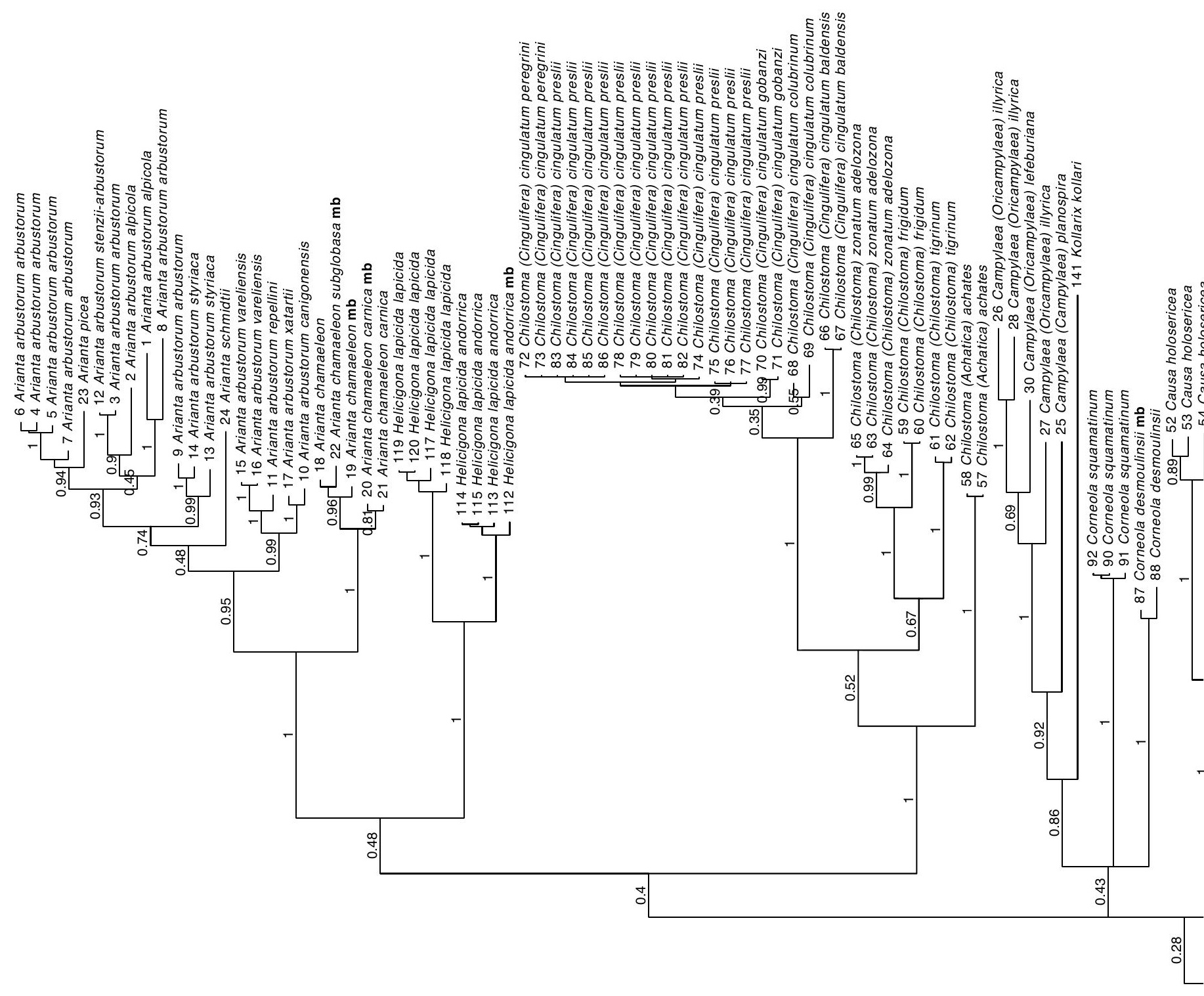




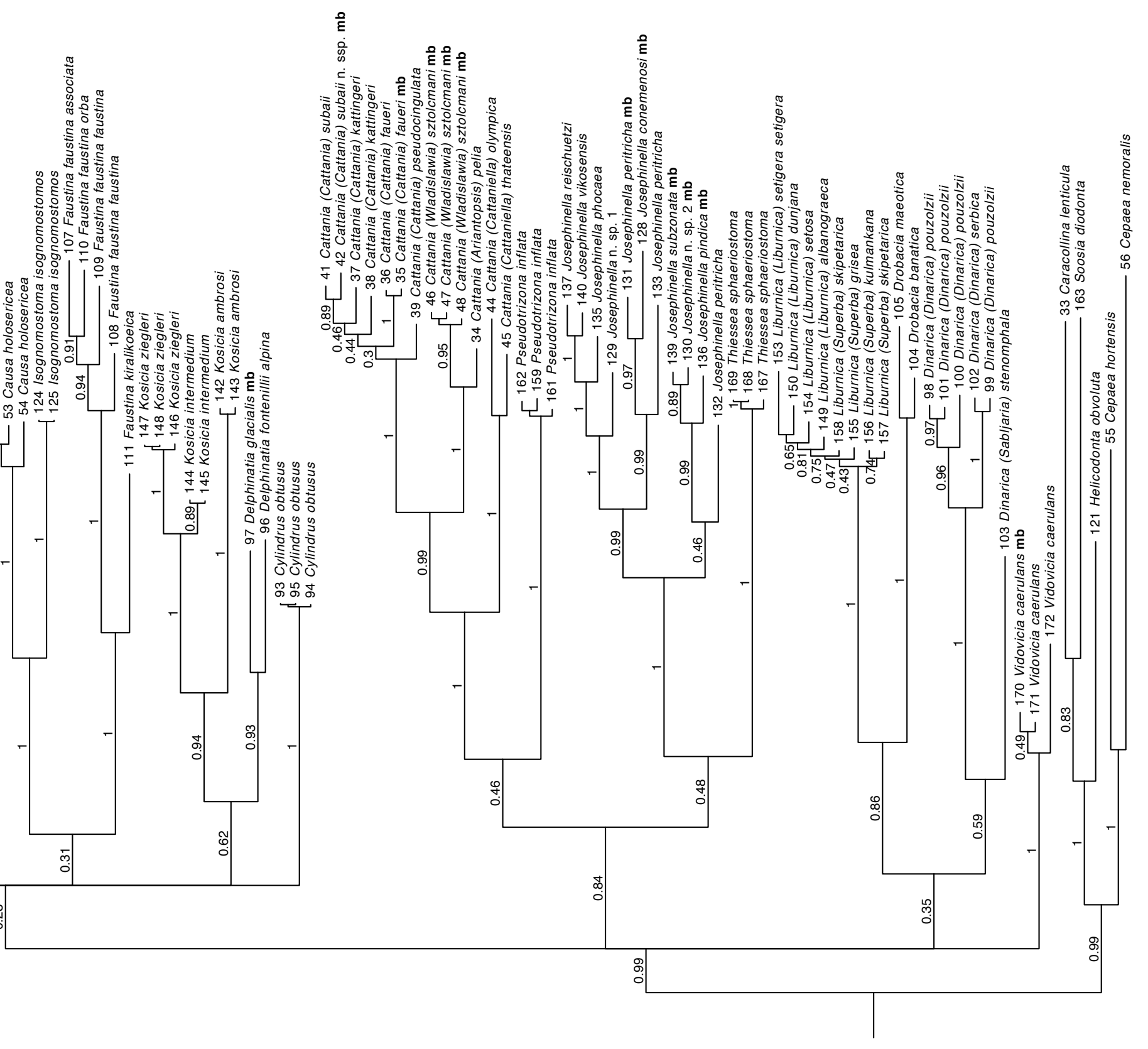


S

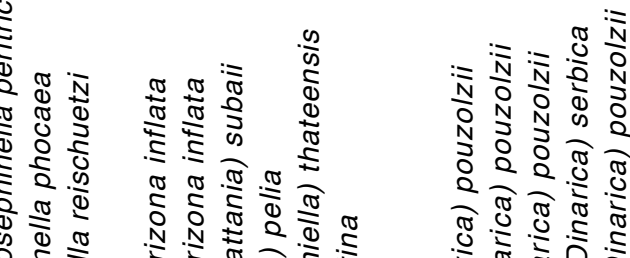

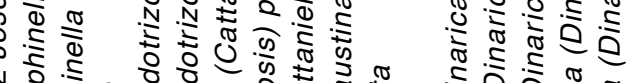

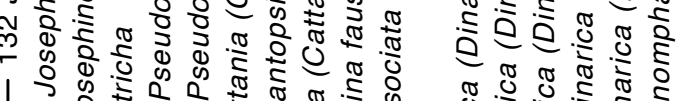

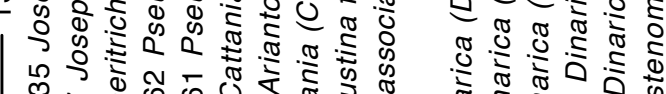

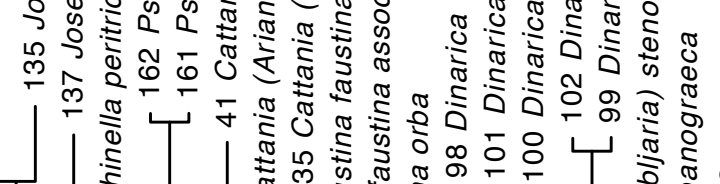

กิ

(5)

:

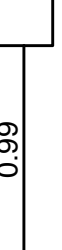

$\stackrel{\infty}{\infty}$

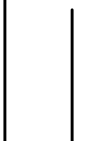
,

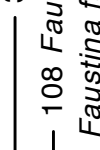
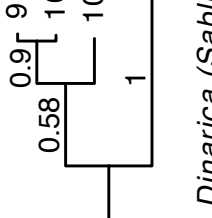

\section{要}

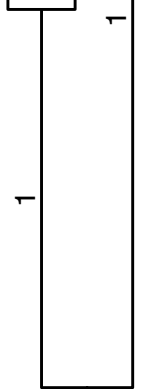

.

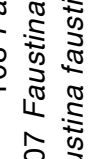

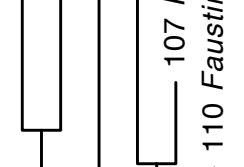

: ग्ञ

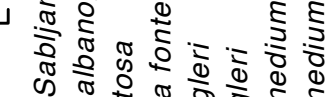

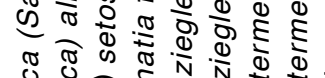

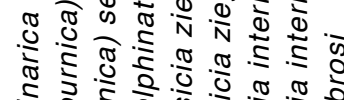

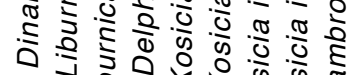

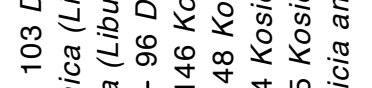

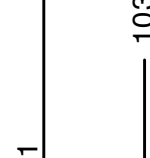




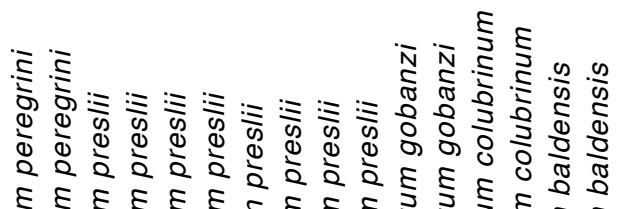

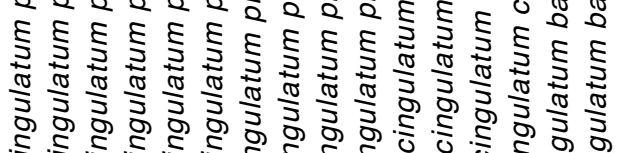

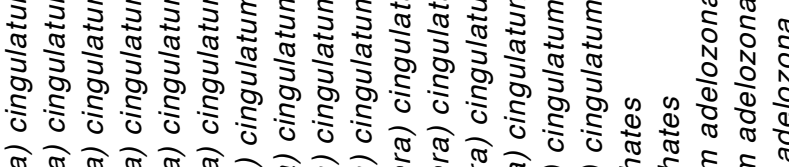

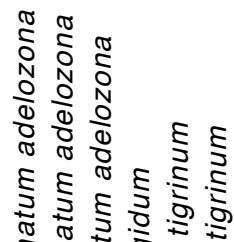

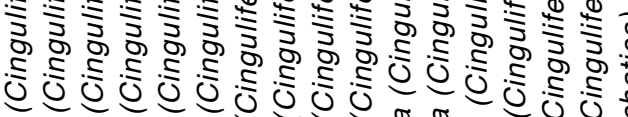
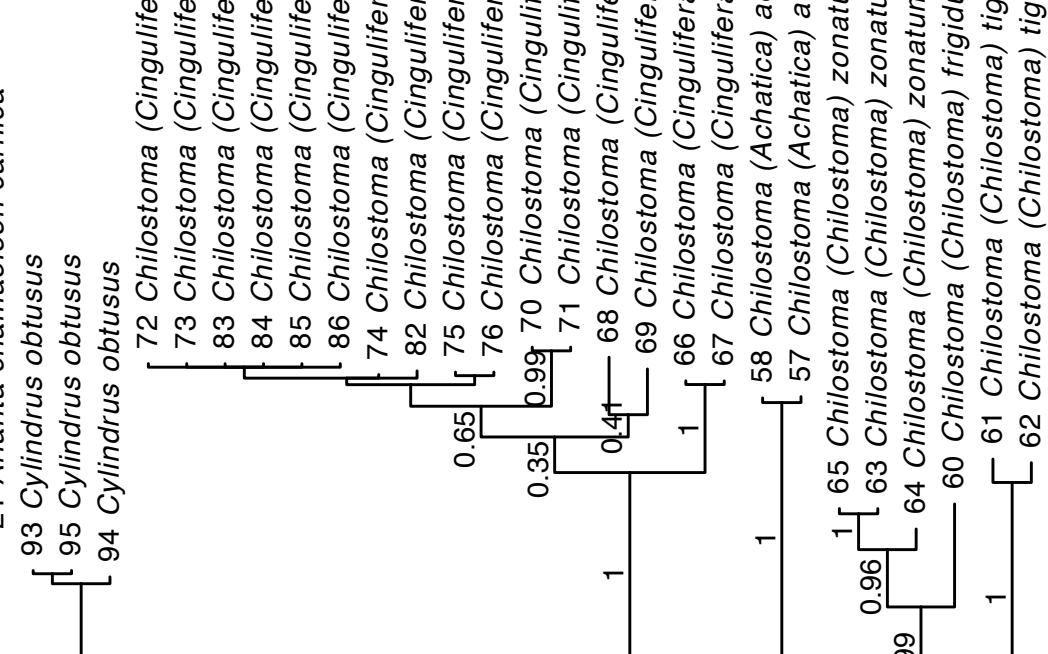
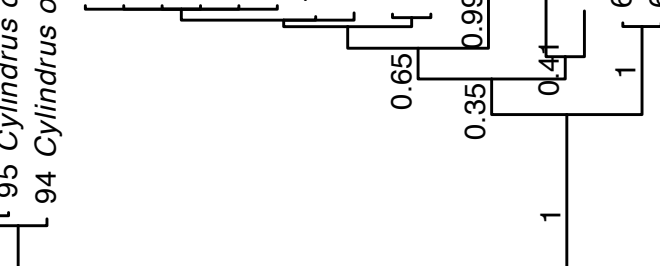


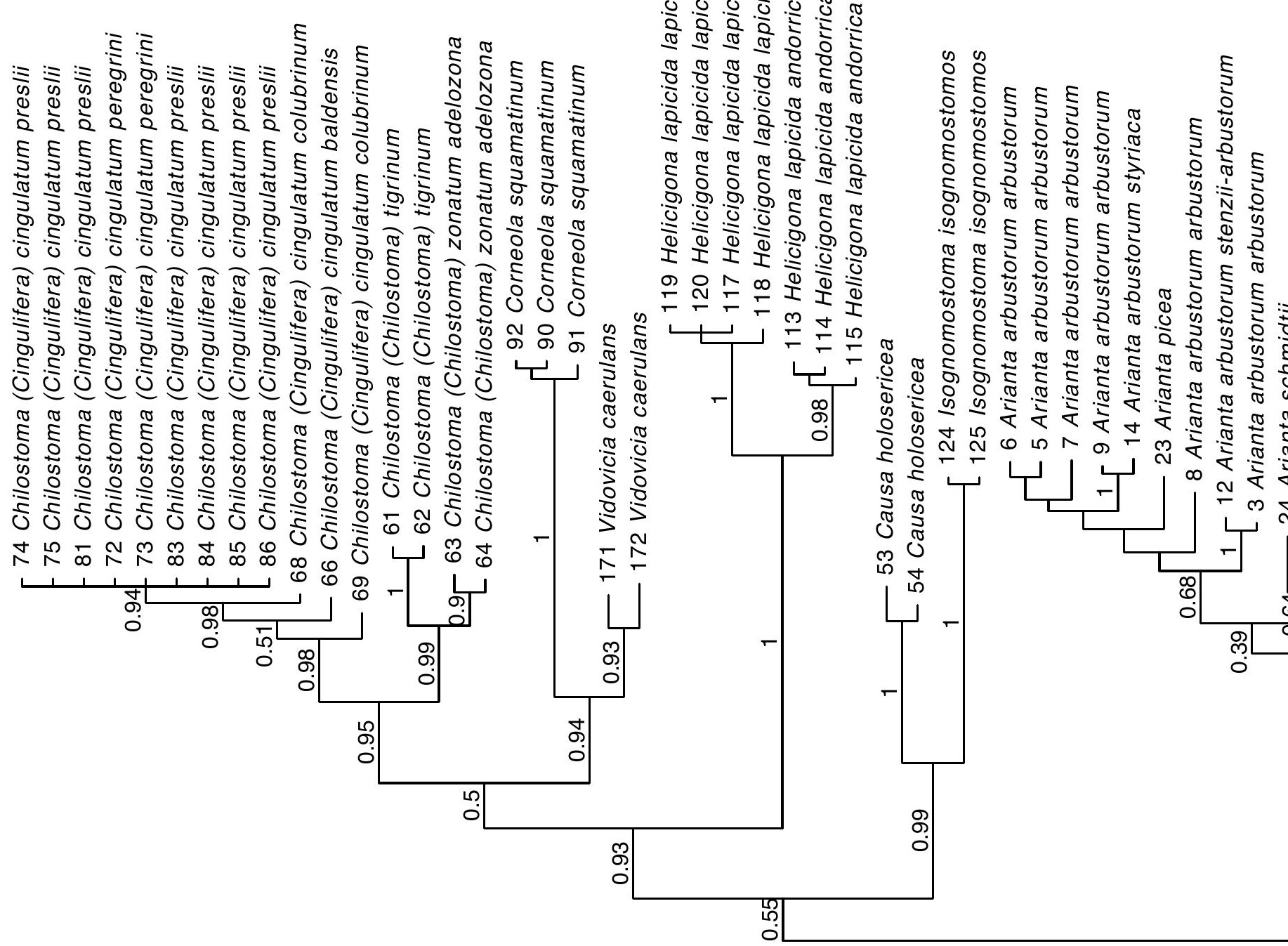




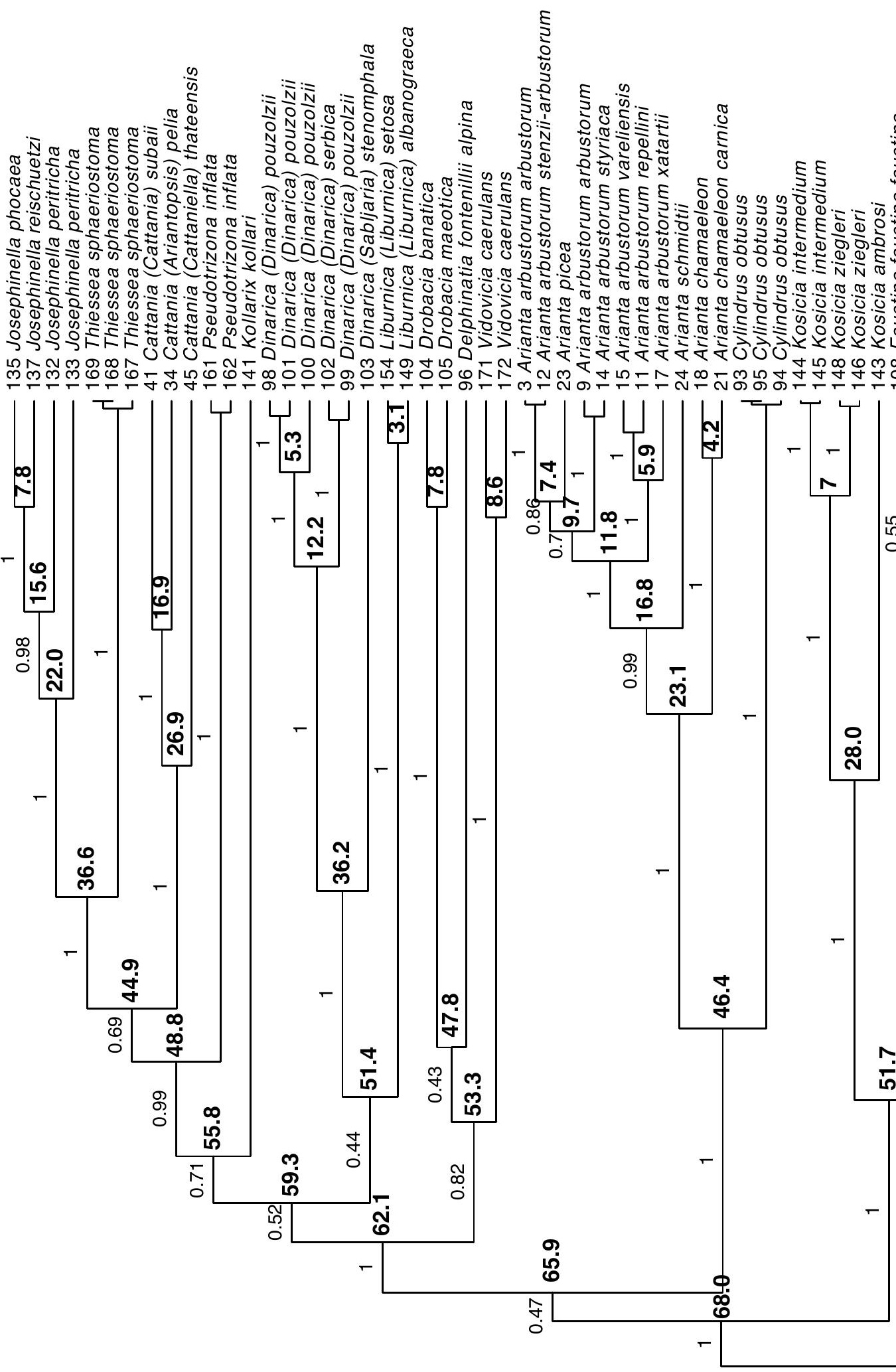




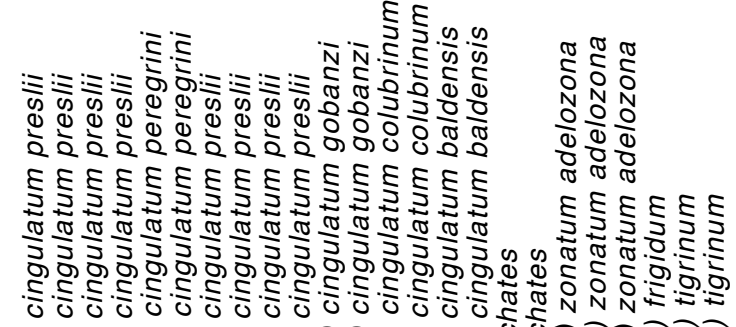

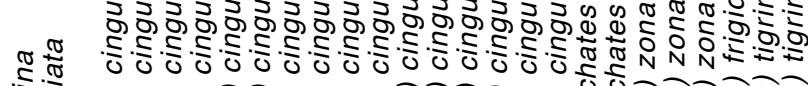

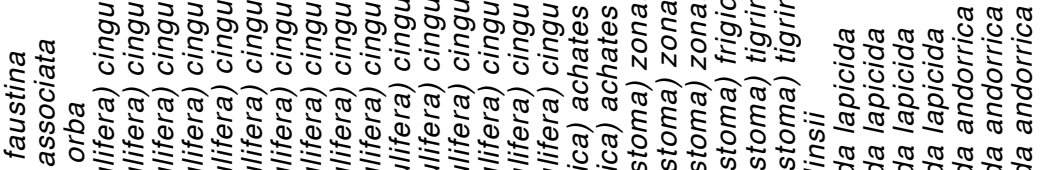

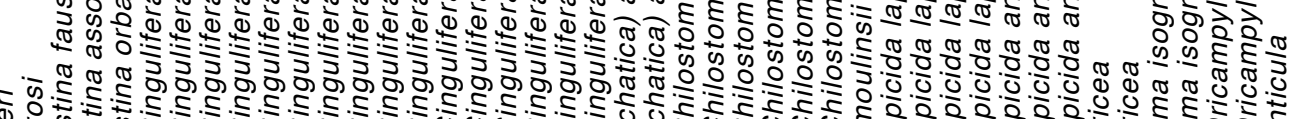

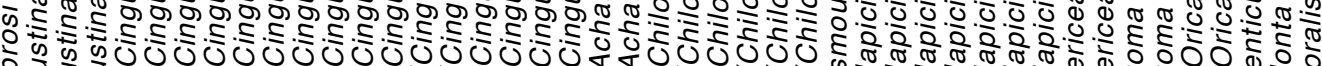
बह

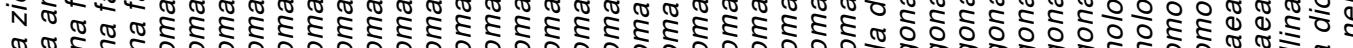

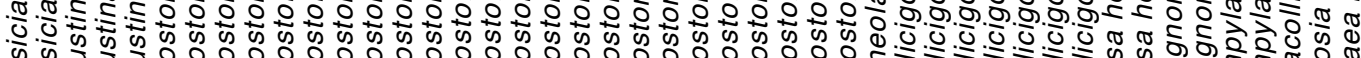

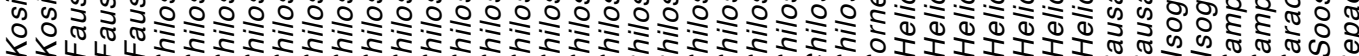

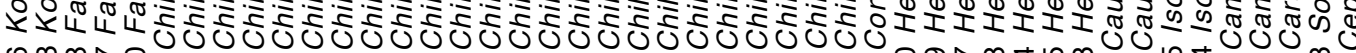

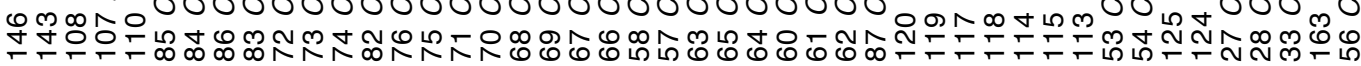

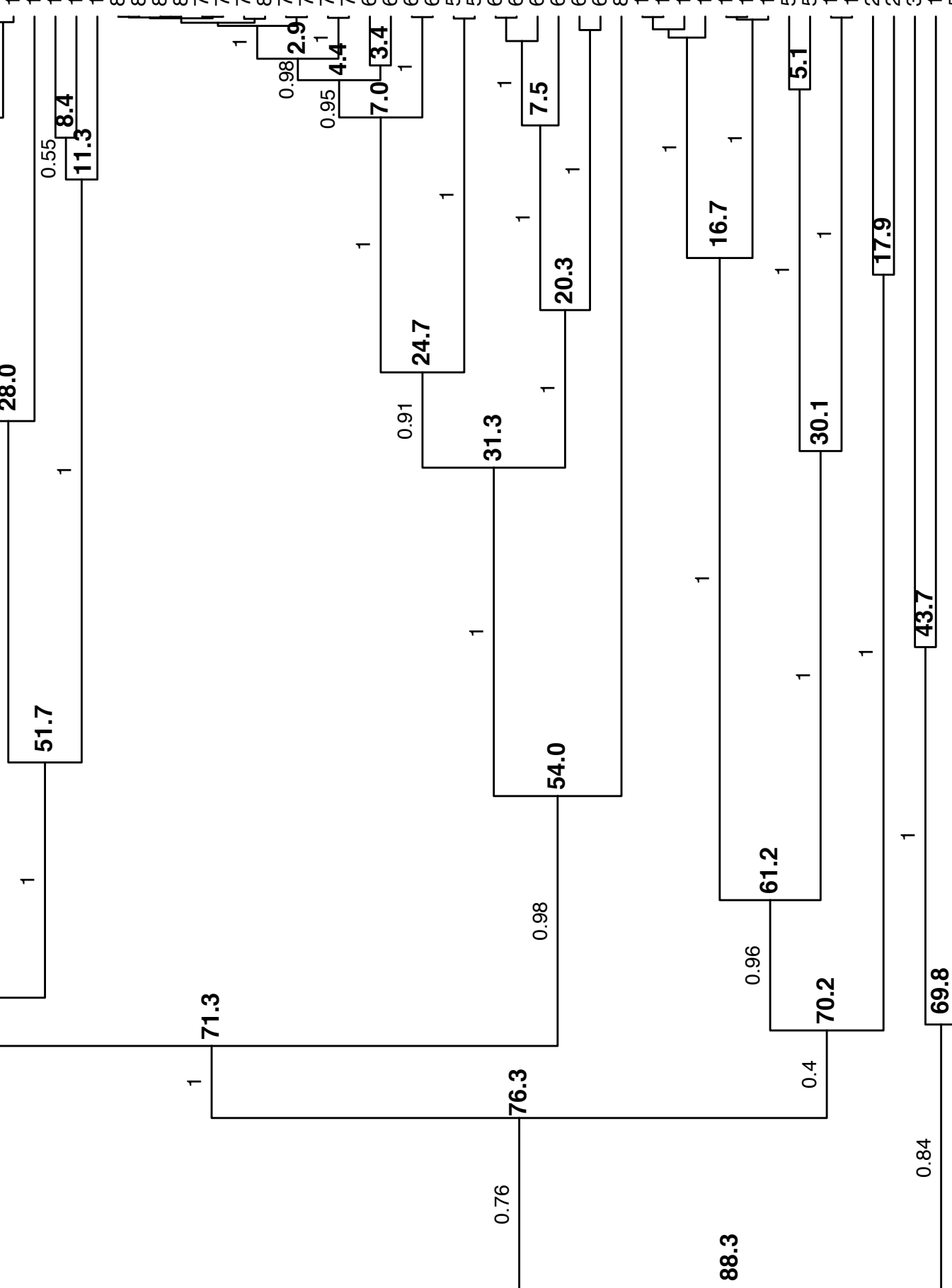




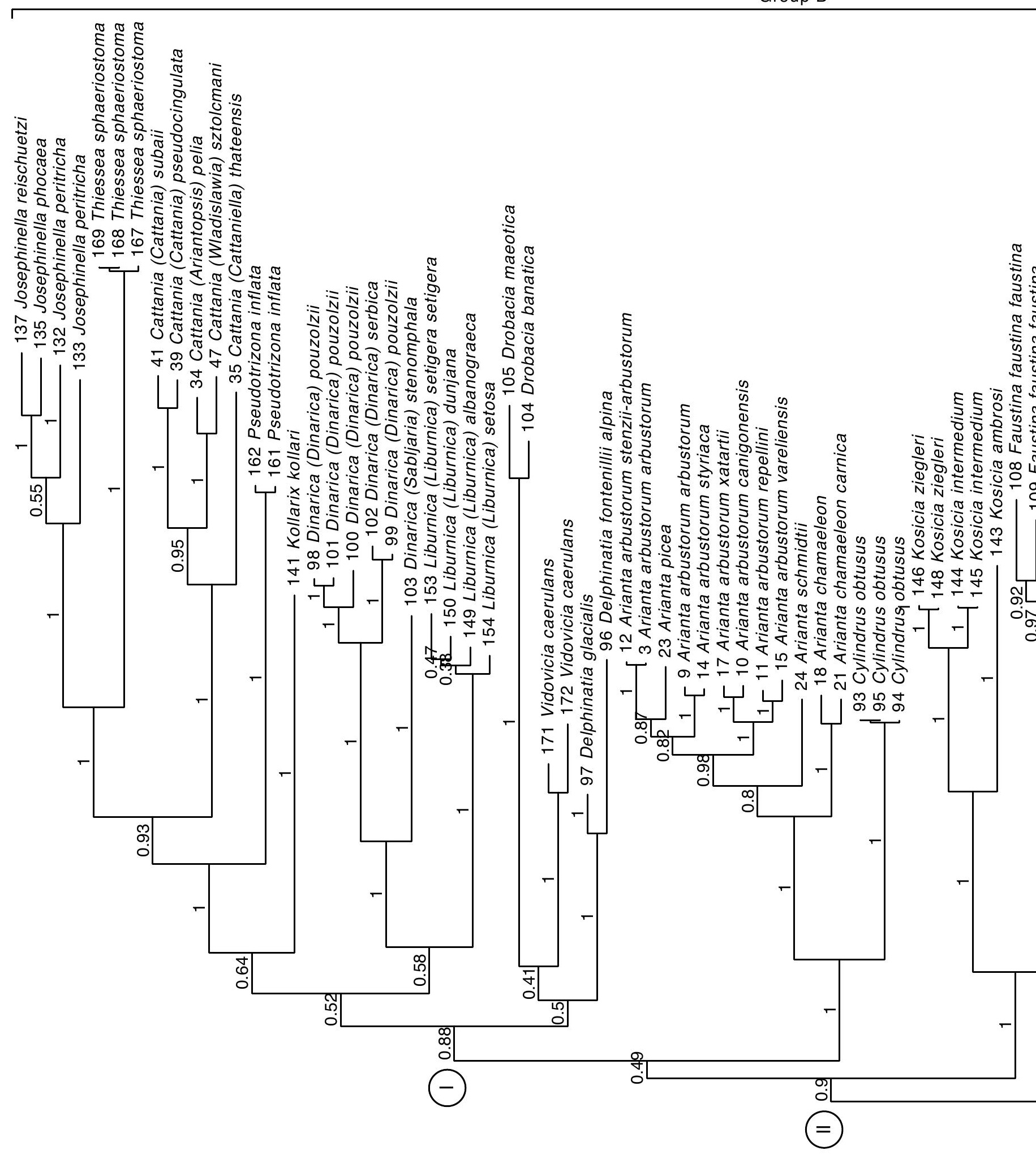




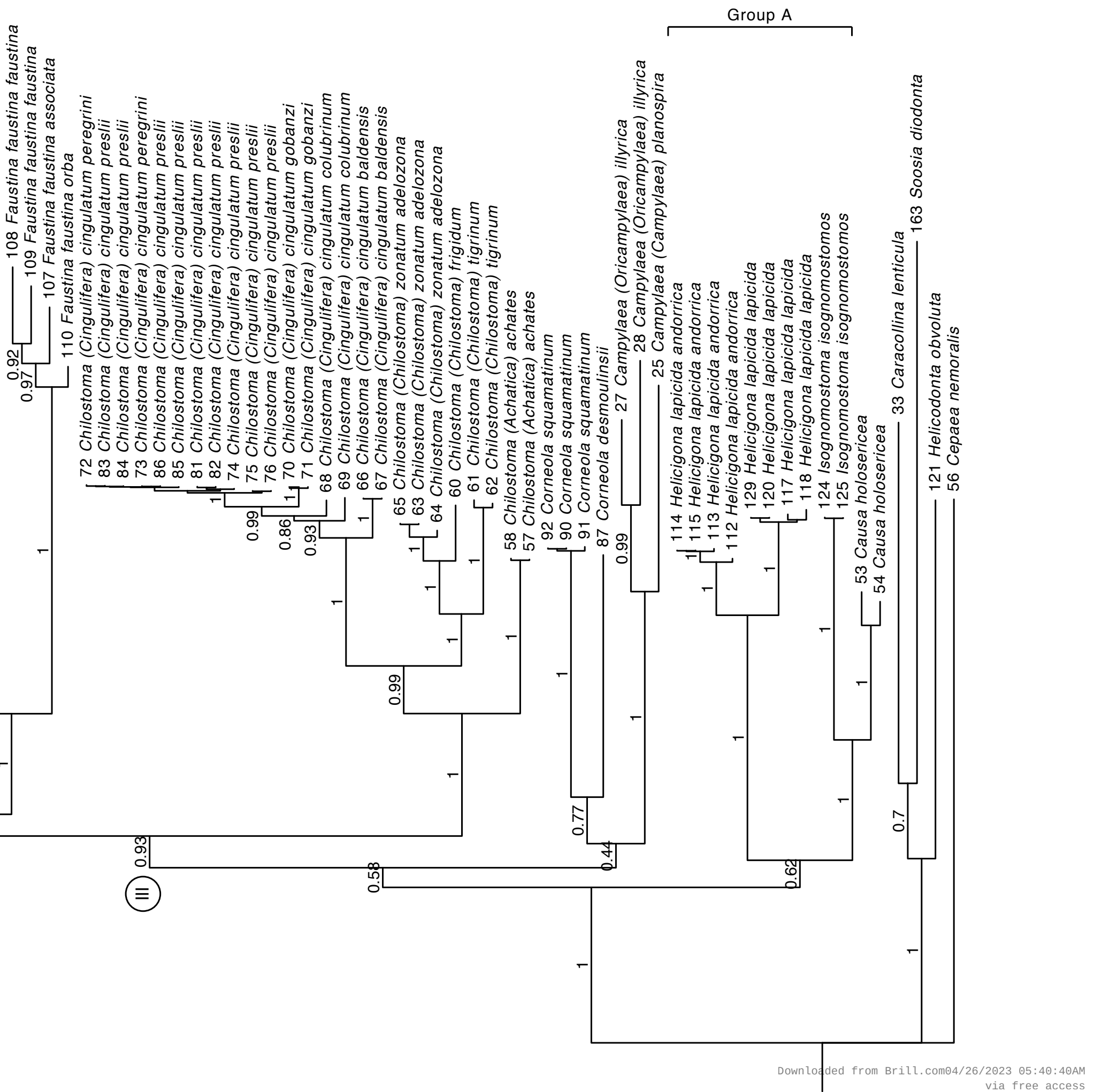

